\title{
A method for computing Stokes flow interactions among spherical objects and its application to suspensions of drops and porous particles
}

Guobiao Mo and Ashok S. Sangani

Citation: Phys. Fluids 6, 1637 (1994); doi: 10.1063/1.868227

View online: http://dx.doi.org/10.1063/1.868227

View Table of Contents: http://pof.aip.org/resource/1/PHFLE6/v6/i5

Published by the American Institute of Physics.

\section{Related Articles}

Criticality of flow transition behind two side-by-side elliptic cylinders

Phys. Fluids 24, 034102 (2012)

Frictional slip lengths for unidirectional superhydrophobic grooved surfaces

Phys. Fluids 23, 072001 (2011)

A study of start-up flow of thixotropic fluids including inertia effects on an inclined plane

Phys. Fluids 23, 013103 (2011)

A formula for the wall-amplified added mass coefficient for a solid sphere in normal approach to a wall and its application for such motion at low Reynolds number

Phys. Fluids 22, 123303 (2010)

Axial pressure-difference between far-fields across a sphere in viscous flow bounded by a cylinder

Phys. Fluids 22, 103305 (2010)

\section{Additional information on Phys. Fluids}

Journal Homepage: http://pof.aip.org/

Journal Information: http://pof.aip.org/about/about_the_journal

Top downloads: http://pof.aip.org/features/most_downloaded

Information for Authors: http://pof.aip.org/authors

\section{ADVERTISEMENT}

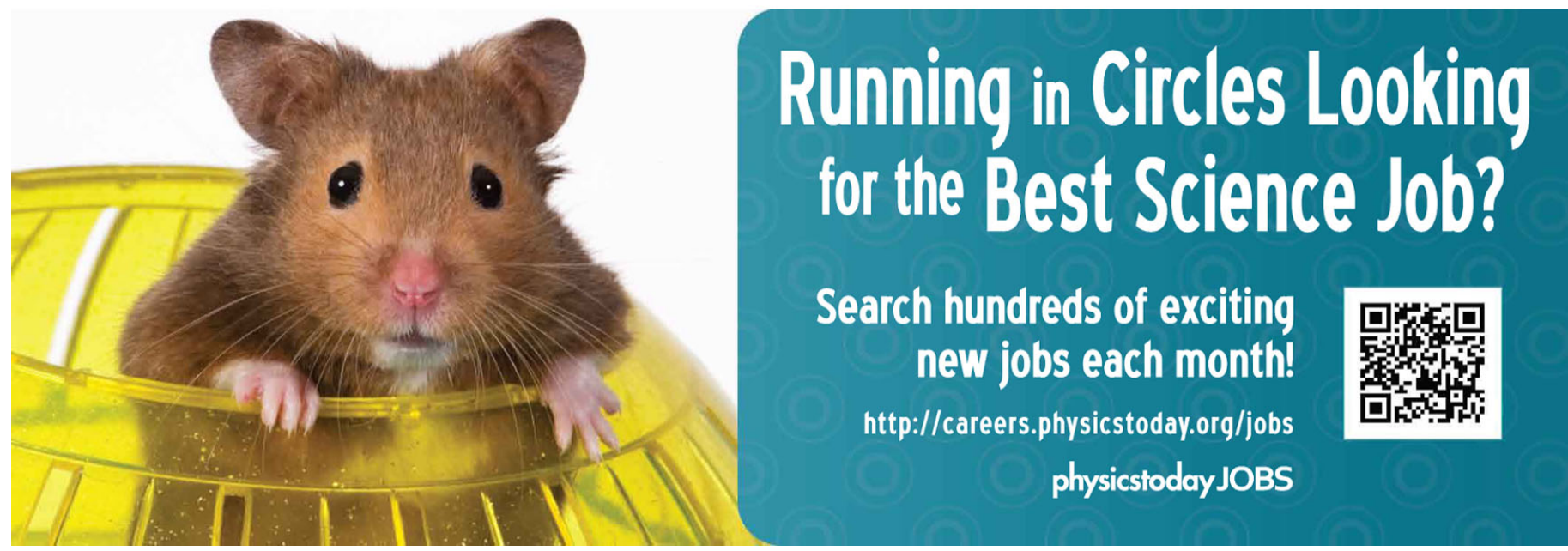




\title{
A method for computing Stokes flow interactions among spherical objects and its application to suspensions of drops and porous particles
}

\author{
Guobiao Mo and Ashok S. Sangani \\ Department of Chemical Engineering and Materials Science, Syracuse University, Syracuse, \\ New York 13244
}

(Received 1 October 1993; accepted 29 December 1993)

\begin{abstract}
A method for computing Stokes flow interactions in suspensions of spherical objects is described in detail and applied to the suspensions of porous particles, drops, and bubbles to determine their hydrodynamic transport coefficients.
\end{abstract}

\section{INTRODUCTION}

The problem of computing Stokes flow interactions among randomly placed particles has been treated by a number of investigators. ${ }^{1-11}$ In particular, Ladd $^{9-11}$ has developed a method based on multipole expansion that accounts for these interactions quite accurately. While, in principle, his method can be extended to treat nonrigid spherical particles as well, this has not been done to date. The purpose of this paper is to present a method we have found suitable for determining the Stokes flow interactions among spherical objects (drops, bubbles, porous or charged particles). Although this method is also based on the multipole expansion, the details are quite different from those of Ladd's method.

The Stokes flow interaction problems can be generally classified into two groups. The first corresponds to determining the macroscopic quantity of interest given the spatial and size distribution of particles, and the second to determining the microstructure of the suspension for a given macroscopic (or imposed) flow and the microscale physics. Naturally, the second group of problems are computationally more intensive as one must carry out dynamic simulations which involve solving for interactions among many particles tens of thousands of times, and it is desirable therefore to devise efficient numerical methods, perhaps at the slight expense of accuracy, in determining the interactions among the particles. For this reason, and because of the poor ability of the method of multipole expansion to account for lubrication forces between closely moving particles, Brady and co-workers ${ }^{6-8}$ have devised an ingenious approximation to the many-body resistivity tensor in terms of a far-field many-body mobility tensor inverse and a close-field pair-additive resistivity tensor. Since the problems falling in the first group can now be solved with a modest computational effort, the method described here is particularly aimed at solving these problems. In a companion paper, we present an alternative to the method used by Brady and Bossis for incorporating the lubrication effects.

\section{THE METHOD}

Let us consider Stokes flow around $N$ spherical (rigid or otherwise) particles placed within a unit cell. The entire medium consists of periodic extensions of this cell. The method of multipole expansion starts with the Green's function $u_{i}^{*}$ for the Stokes equation, which corresponds to the spatially periodic solution of the Stokes equation with point forces at the lattice points of the periodic array, i.e.,

$$
\left(\eta \nabla^{2} u_{i}^{*}-\partial_{i} p^{*}\right)(\mathbf{x})=\partial_{j} \sigma_{i j}^{*}=\sum_{\mathbf{x}_{\mathbf{L}}} F_{i} \delta\left(\mathbf{x}-\mathbf{x}_{\mathbf{L}}\right), \quad \partial_{i} u_{i}^{*}=0,
$$

where $\mathbf{x}_{\mathbf{L}}$ denotes the lattice points, $F_{i}$ the force, $\sigma_{i j}^{*}$ the stress tensor, and $p^{*}$ the pressure in the fluid. Throughout the paper, we shall use the Einstein summation convention and an abbreviated notation for partial differentiation. Thus, $\partial_{i} \equiv \partial / \partial x_{i}, \partial^{2}{ }_{i j} \equiv \partial^{2} / \partial x_{i} \partial x_{j}$, etc. A solution of (1) is given by ${ }^{12}$

$$
\begin{aligned}
& \qquad \begin{aligned}
u_{i}^{*}(\mathbf{x}) & =A_{j} v_{i j}(\mathbf{x}), \quad v_{i j}=S_{1} \delta_{i j}-\partial^{2}{ }_{i j} S_{2}, \\
p^{*}(\mathbf{x}) & =\eta A_{j}\left(4 \pi x_{j} / \tau-\partial_{j} S_{1}\right),
\end{aligned} \\
& \text { with } A_{j}=-F_{j} /(4 \pi \eta) \text {, and }
\end{aligned}
$$

$$
\begin{gathered}
S_{m}(\mathbf{x})=\frac{\left(-4 \pi^{2}\right)^{1-m}}{\pi \tau} \sum_{\mathbf{k} \neq 0} k^{-2 m} \exp (-2 \pi i \mathbf{k} \cdot \mathbf{x}), \\
m=1,2 .
\end{gathered}
$$

Here, $\tau$ is the volume of the unit cell and $\mathbf{k}$ the vectors in the reciprocal lattice.

Now an infinite number of spatially periodic solutions of the Stokes equation are generated by successive differentiation of the Green's function given by (2) and a general solution to the Stokes equation is obtained by superposition. Since the velocity is regular at all points in the fluid, we must superimpose these solutions such that the singularities are all inside the particles. Thus, we write the general solution as

$$
u_{i}(\mathbf{x})=U_{i}^{\infty}(\mathbf{x})+\sum_{\alpha=1}^{N} \mathscr{H}_{j}^{\alpha} v_{i j}\left(\mathbf{x}-\mathbf{x}^{\alpha}\right),
$$

where $U_{i}^{\infty}$ is the imposed macroscopic flow, and $\mathscr{M}_{j}^{\alpha}$ the differential operator given by

$$
\mathscr{M}_{j}^{\alpha}=A_{j}^{\alpha}+B_{j k}^{\alpha} \partial_{k}+C_{j k l}^{\alpha} \partial_{k l}^{2}+\cdots .
$$

The constants $A_{j}^{\alpha}, B_{j k}^{\alpha}$, etc., are referred to as the force monopole, dipole, etc., and are to be determined from the boundary conditions on the surface of the particle to complete the solution. The above formal solution with a modified definition of $\mathscr{M}_{j}^{\alpha}$ in terms of irreducible forcemoments tensors has been used by Mazur and 
co-workers ${ }^{1-4}$ and Ladd. ${ }^{9-11}$ As evident from these studies, the subsequent analysis can be quite cumbersome even for the simple case of rigid particles due to the tensorial nature of the force multipoles. Our method departs from these studies by redefining $\mathscr{M}_{j}^{\alpha}$ in terms of constants appearing in the Lamb's general solution ${ }^{13,14}$ for Stokes flow around spherical objects.

The Lamb's general solution around particle $\alpha$ is written as

$\mathbf{u}^{\alpha}(\mathbf{x})=\sum_{n=-\infty}^{\infty}\left[\left(c_{n} r^{2} \nabla p_{n}^{\alpha}+b_{n} \mathbf{r} p_{n}^{\alpha}\right)+\nabla \times\left(\mathbf{r} \chi_{n}^{\alpha}\right)+\nabla \phi_{n}^{\alpha}\right]$,

with $\mathbf{r}=\mathbf{x}-\mathbf{x}^{\alpha}$ and

$$
c_{n}=\frac{n+3}{2(n+1)(2 n+3)}, \quad b_{n}=\frac{-n}{(n+1)(2 n+3)} .
$$

Here, $p_{n}^{\alpha}$ is related to the pressure by $p=\eta \Sigma p_{n}^{\alpha}$, and $p_{n}^{\alpha}$, $\chi_{n}^{\alpha}$, and $\phi_{n}^{\alpha}$ are the $n$ th-order spherical harmonics. The harmonics of negative order are singular at $\mathbf{x}=\mathbf{x}^{\alpha}$, or at $r=0$, and we express them by

$$
\begin{aligned}
& p_{-n-1}^{\alpha}=\sum_{m=0}^{n}\left(P_{n m}^{\alpha} Y_{n}^{m}+\widetilde{P}_{n m}^{\alpha} \widetilde{Y}_{n}^{m}\right) r^{-n-1}, \\
& \chi_{-n-1}^{\alpha}=\sum_{m=0}^{n}\left(T_{n m}^{\alpha} Y_{n}^{m}+\tilde{T}_{n m}^{\alpha} \tilde{Y}_{n}^{m}\right) r^{-n-1},
\end{aligned}
$$

with a similar expression for $\phi_{-n-1}^{\alpha}$ in terms of constants $\Phi_{n m}^{\alpha}$ and $\widetilde{\Phi}_{n m}^{\alpha}$. Here, $Y_{n}^{m}$ and $\widetilde{Y}_{n}^{m}$ are the surface harmonics

$$
Y_{n}^{m}=P_{n}^{m}(\cos \theta) \cos m \varphi, \quad \widetilde{Y}_{n}^{m}=P_{n}^{m}(\cos \theta) \sin m \varphi,
$$

where $P_{n}^{m}$ is the associated Legendre function, and $\theta$ and $\varphi$ the spherical polar angles measured with respect to the coordinate system centered at $\mathbf{x}^{\alpha}$ with the $x_{1}$-axis as the polar axis. Since the leading singularity due to a point force corresponds to $n=-2$ in (7), the term with $n=-1$ must be discarded from (7) except for $\phi_{-1}$, which must be retained whenever the particles act as a source or sink of fluid, e.g. in applications involving a phase change at the boundary of the particles.

The harmonics with non-negative $n$ are regular at $r=0$, and we write

$$
\eta^{-1} p^{\alpha, r}=\sum_{n=0}^{\infty} p_{n}^{\alpha}=\sum_{n=0}^{\infty} \sum_{m=0}^{n}\left(P_{n m}^{\alpha, r} Y_{n}^{m}+\tilde{P}_{n m}^{\alpha, r} \tilde{Y}_{n}^{m}\right) r^{n},
$$

with similar expressions for $\phi^{\alpha, r}$ and $\chi_{n}^{\alpha, r}$ in terms of $\Phi_{n m}^{\alpha, r}, \widetilde{\Phi}_{n m}^{\alpha, r}$ and $T_{n m}^{\alpha, r}, \widetilde{T}_{n m}^{\alpha, r}$, respectively. Now most boundary conditions on the spherical surfaces can be applied readily to the velocity field given by (7) to obtain a set of relatively simple expressions among the 12 coefficients $P_{n m}^{\alpha}, P_{n m}^{\alpha, r}$, etc., defined above, and thus the main problem we need to address here is the relations between these coefficients and the constants that appear in the differential operators such as (6).
The singular terms in (7) can arise only from the singularities in $v_{i j}\left(\mathbf{x}-\mathbf{x}^{\alpha}\right)$. As $r=\left|\mathbf{x}-\mathbf{x}^{\alpha}\right| \rightarrow 0, S_{1} \rightarrow 1 / r$, and $S_{2} \rightarrow$ const $+r / 2$ (see Ref. 12), and therefore, the singular part of $v_{i j}$ is

$$
v_{i j}^{\alpha, s}=\delta_{i j} r^{-1}-\partial_{i j}^{2}(r / 2)=\left(r_{i} r_{j}+r^{2} \delta_{i j}\right) /\left(2 r^{3}\right),
$$

the Oseen tensor for the flow due to a point force in a fluid at rest at infinity. The derivatives of this tensor as required by (6) are rather complicated to evaluate and, therefore, as mentioned earlier, we modify the definition of the differential operator. In particular, we shall redefine $\mathscr{M}_{j}^{\alpha}$ such that

$$
\mathscr{M}_{j}^{\alpha} v_{i j}^{\alpha, s} \equiv u_{i}^{\alpha, s},
$$

$u_{i}^{\alpha, s}$ being the singular part of the velocity defined by the summation over $n$ from $-\infty$ to -2 in (7).

To begin redefining the differential operator, we first quote some results of differentiation taken from Hobson ${ }^{15}$ and Sangani and Acrivos: ${ }^{16}$

$$
\begin{aligned}
& \mathscr{D}_{n}^{m} r^{-1}=\lambda_{n m} Y_{n}^{m} r^{-n-1}, \\
& \mathscr{D}_{n}^{m} r=\frac{\lambda_{n m}}{(2 n-1)}\left[Y_{n-2}^{m}-Y_{n}^{m}\right] r^{1-n}, \\
& \widetilde{D}_{n}^{m} r^{-1}=\lambda_{n m} \tilde{Y}_{n}^{m} r^{-n-1}, \\
& \mathscr{D}_{n}^{m} r=\frac{\lambda_{n m}}{(2 n-1)}\left[\widetilde{Y}_{n-2}^{m}-\tilde{Y}_{n}^{m}\right] r^{1-n},
\end{aligned}
$$

where

$$
\begin{aligned}
& \lambda_{n m}=(-1)^{n-m} 2^{1-m}(n-m) !, \\
& \mathscr{D}_{n}^{m}=\partial_{1}^{n-m} \Delta_{m}, \widetilde{\mathscr{D}}_{n}^{m}=\partial_{1}^{n-m}{\widetilde{\Delta_{m}}}_{m},
\end{aligned}
$$

with

$$
\begin{aligned}
& \Delta_{m}=\partial_{\xi}^{m}+\partial_{\eta}^{m}, \quad \widetilde{\Delta}_{m}=i\left[\partial_{\xi}^{m}-\partial_{\eta}^{m}\right], \\
& \xi=x_{2}+i x_{3}, \quad \eta=x_{2}-i x_{3} .
\end{aligned}
$$

The reason for using such awkward looking differential operators is that, when operated on $r^{-1}$, they produce a single spherical harmonic. In contrast, a differentiation of $r^{-1}$ with the $n$th term in (6) will, in general, produce a sum of $n-1$ harmonics.

Since the differential operators $\mathscr{D}_{n}^{m}$ and $\widetilde{\mathscr{D}}_{n}^{m}$ are linearly independent from the Laplacian operator, and since $v_{i j}$ is biharmonic, we separate the Laplacian component, say, $\mathscr{M}_{j}^{L} \nabla^{2}$, from the rest of the differential operator $\mathscr{H}_{j}$. Now using the relations ${ }^{12}$

$$
\nabla^{2} S_{2}=S_{1}, \quad \nabla^{2} S_{1}=4 \pi\left(\tau^{-1}-\sum_{\mathbf{x}_{\mathbf{L}}} \delta\left(\mathbf{x}-\mathbf{x}_{\mathbf{L}}\right)\right),
$$

we see that, inside the fluid, $\nabla^{2} v_{i j}=4 \pi \tau^{-1} \delta_{i j}-\partial^{2}{ }_{i j} S_{1}$, or $\mathscr{H}_{j}^{L} \nabla^{2} v_{i j}=-\mathscr{H}_{j}^{L} \partial^{2}{ }_{i j} S_{1}$ plus a constant. In view of the above then, we rewrite (5) as

$$
\begin{aligned}
u_{i}(\mathbf{x})= & u_{i}^{\infty}(\mathbf{x})+\sum_{\alpha=1}^{N}\left[\mathscr{G}_{j}^{\alpha}\left(\delta_{i j} S_{1}-\partial_{i j}^{2} S_{2}\right)\left(\mathbf{x}-\mathbf{x}^{\alpha}\right)\right. \\
& \left.+\mathscr{H}^{\alpha} \partial_{j} S_{1}\left(\mathbf{x}-\mathbf{x}^{\alpha}\right)\right],
\end{aligned}
$$


where $\mathscr{H} \equiv-\partial_{j} \mathscr{M}_{j}^{L}$. There is a slight difference between $U_{i}^{\infty}$ defined in (5) and $u_{i}^{\infty}$ in the above expression that arises because of the constant term in $\nabla^{2} S_{1}$. The exact relationship is derived later [cf. (69)]. The differential operators $\mathscr{G}^{\alpha}$ and $\mathscr{H}^{\alpha}$ are given by

$$
\begin{aligned}
\mathscr{Y}_{j}^{\alpha} & =\sum_{n=0}^{\infty} \sum_{m=0}^{n}\left[G_{j n m}^{\alpha} \mathscr{D}_{n}^{m}+\widetilde{G}_{j n m}^{\alpha} \mathscr{\mathscr { D }}_{n}^{m}\right], \\
\mathscr{H}^{\alpha} & =\sum_{n=0}^{\infty} \sum_{m=0}^{n}\left[H_{n m}^{\alpha} \mathscr{D}_{n}^{m}+\widetilde{H}_{n m}^{\alpha} \mathscr{\mathscr { D }}_{n}^{m}\right] .
\end{aligned}
$$

The number of unknowns in the formal solution (20)(21) is more than the minimum necessary as can be seen by comparing it with the Lamb's solution (7). This is because some of the terms in the operator $\mathscr{G}^{\alpha}$ are linearly related to the others through the equation of continuity. Thus, our first task will be to reduce the unknowns by expressing $\mathscr{G}^{\alpha}$ in terms of the coefficients of the singular terms in (20) at $\mathbf{x}^{\alpha}$. More specifically, we rewrite (13) as

$$
\begin{aligned}
u_{i}^{\alpha, s} & \equiv \mathscr{G}{ }_{j}^{\alpha} v_{i j}^{\alpha, s}+\mathscr{H}^{\alpha} \partial_{i} r^{-1} \\
& \equiv \sum_{n=-\infty}^{-2}\left[\left(c_{n} r^{2} \nabla p_{n}^{\alpha}+b_{n} \mathbf{r} p_{n}^{\alpha}\right)+\nabla \times\left(\mathbf{r} \chi_{n}^{\alpha}\right)+\nabla \phi_{n}^{\alpha}\right]
\end{aligned}
$$

To determine the form of $\mathscr{G}^{\alpha}$ that satisfies the above requirement, we shall decompose it into two parts $\mathscr{G}^{\alpha, T}$ and $\mathscr{G}^{\alpha, P}$ with the former giving terms when operated on $v_{i j}^{s}$ that correspond to those containing the poloidal components $\chi_{n}^{\alpha}$ in (22) and the latter to $p_{n}^{\alpha}$. Finally, the operator $\mathscr{H}^{\alpha}$ will be chosen such that the remaining terms correspond to $\phi_{n}^{\alpha}$.

We begin with the determination of $\mathscr{G}^{\alpha, T}$. Since $\chi_{n}^{\alpha}$ is the only part of the velocity field related to the radial component of the vorticity, we first determine the vorticity in the fluid by taking the curl of $(20)$ :

$$
\omega(\mathbf{x})=\nabla \times \mathbf{u}^{\infty}(\mathbf{x})+\sum_{\alpha=1}^{N}\left(\nabla \times \mathscr{G}^{\alpha}\right) S_{1}\left(\mathbf{x}-\mathbf{x}^{\alpha}\right) .
$$

The singular part of $S_{1}$ is $1 / r$ and therefore we define $\mathscr{G}^{\alpha, T}$ via

$$
\mathscr{G}^{\alpha, T^{-1}} \equiv \sum_{n=-\infty}^{-2} \nabla \times\left(\mathbf{r} \chi_{n}^{\alpha}\right)
$$

Substituting for $\chi_{n}^{\alpha}$ from (9) into (24), using $Y_{n m} r^{-n-1}=\lambda_{n m}^{-1} \mathscr{D}_{n}^{m} r^{-1}$ [cf. (14)], and comparing the resulting expression with (21), we find that

$$
\mathscr{G}_{1}^{\alpha, T}=\sum_{n=1}^{\infty} \sum_{m=0}^{n} \lambda_{n m}^{-1} m\left[-\widetilde{T}_{n m}^{\alpha} \mathscr{D}_{n}^{m}+T_{n m}^{\alpha} \mathscr{\mathscr { D }}_{n}^{m}\right] .
$$

In a similar manner we determine the other two components of $\mathscr{F}_{j}^{\alpha, T}$ :

Phys. Fluids, Vol. 6, No. 5, May 1994

$$
\begin{aligned}
\mathscr{G}_{2}^{\alpha, T}= & \sum_{n=1}^{\infty} \sum_{m=0}^{n} \lambda_{n m}^{-1}\left((n-m)\left(1+\delta_{m 0}\right)\right. \\
& \times\left(\widetilde{T}_{n m}^{\alpha} \mathscr{D}_{n}^{m+1}-T_{n m}^{\alpha} \widetilde{\mathscr{D}}_{n}^{m+1}\right)+\frac{n+m}{4}\left(1-\delta_{m 0}\right) \\
& \left.\times\left(\widetilde{T}_{n m}^{\alpha} \mathscr{D}_{n}^{m-1}-T_{n m}^{\alpha} \widetilde{\mathscr{D}}_{n}^{m-1}\right)\right) \\
\mathscr{Y}_{3}^{\alpha, T}= & \sum_{n=1}^{\infty} \sum_{m=0}^{n} \lambda_{n m}^{-1}\left((n-m)\left(1+\delta_{m 0}\right)\right. \\
& \times\left(T_{n m}^{\alpha} \mathscr{D}_{n}^{m+1}+\widetilde{T}_{n m}^{\alpha} \mathscr{D}_{n}^{m+1}\right)-\frac{n+m}{4}\left(1-\delta_{m 0}\right) \\
& \left.\times\left(T_{n m}^{\alpha} \mathscr{D}_{n}^{m-1}+\widetilde{T}_{n m}^{\alpha} \widetilde{\mathscr{D}}_{n}^{m-1}\right)\right) .
\end{aligned}
$$

Now we proceed to determine $\mathscr{G}^{\alpha, P}$ by matching the singular terms related to the pressure. Substituting (20) into the Stokes equation [cf. (1)], and integrating, we obtain the following expression for the pressure in the fluid

$p(\mathbf{x})=\eta \sum_{\alpha=1}^{N}\left[\mathbf{x} \cdot \mathscr{G}^{\alpha} \nabla^{2} S_{1}\left(\mathbf{x}-\mathbf{x}^{\alpha}\right)-\nabla \cdot \mathscr{G}^{\alpha} S_{1}\left(\mathbf{x}-\mathbf{x}^{\alpha}\right)\right]$,

where we have assumed that $\nabla^{2} \mathbf{u}^{\infty}=0$, as is usually the case. In writing the first term on the right-hand side of the above equation, we made use of the fact that $\nabla^{2} S_{1}$ is constant for all points in the fluid [cf. (19)] and thus this part contributes to an overall pressure gradient. Now, since $\nabla \cdot \mathscr{G}^{\alpha, T} r^{-1} \equiv 0$, we choose $\mathscr{G}^{\alpha, P}$ such that $-\nabla \cdot \mathscr{G}^{\alpha, P} r^{-1}$ corresponds to the singular part of the pressure near particle $\alpha$ :

$$
-\nabla \cdot \mathscr{G}^{\alpha, P_{r}}{ }^{-1}=\sum_{n=-\infty}^{-2} p_{n}^{\alpha} .
$$

This and the requirement that the radial component of $\nabla \times \mathscr{G}^{\alpha, P} r^{-1}$ must vanish are satisfied if we choose

$$
\mathscr{G}^{\alpha,} P_{r^{-1}}=\sum_{n=1}^{\infty} \sum_{m=0}^{n} \frac{r^{1-2 n}}{n(2 n-1)} \nabla\left[r^{n}\left(P_{n m}^{\alpha} Y_{n}^{m}+\widetilde{P}_{n m}^{\alpha} \widetilde{Y}_{n}^{m}\right)\right]
$$

Now substituting (21) into (30) and using (14)-(18), it can be shown that the suitable definition of $\mathscr{G}^{\alpha, P}$ is

$$
\begin{aligned}
\mathscr{F}_{1}^{\alpha, P}= & -\sum_{n=1}^{\infty} \sum_{m=0}^{n} \frac{(n-m)(n+m)}{n(2 n-1) \lambda_{n m}} \\
& \times\left[P_{n m}^{\alpha} \widetilde{\mathscr{D}}_{n-1}^{m}+\widetilde{P}_{n m}^{\alpha} \widetilde{D}_{n-1}^{m}\right], \\
\mathscr{F}_{2}^{\alpha, P}= & \sum_{n=1}^{\infty} \sum_{m=0}^{n} \frac{1}{4 n(2 n-1) \lambda_{n m}}\left[4\left(1+\delta_{m 0}\right)(n-m)\right. \\
& \times(n-m-1)\left(P_{n m}^{\alpha} \mathscr{D}_{n-1}^{m+1}+\widetilde{P}_{n m^{\alpha}}^{\alpha} \widetilde{\mathscr{D}}_{n-1}^{m+1}\right)-\left(1-\delta_{m 0}\right) \\
& \left.\times(n+m)(n+m-1)\left(P_{n m}^{\alpha} \mathscr{D}_{n-1}^{m-1}+\widetilde{P}_{n m}^{\alpha} \mathscr{D}_{n-1}^{m-1}\right)\right],
\end{aligned}
$$




$$
\begin{aligned}
\mathscr{G}_{3}^{\alpha, P}= & -\sum_{n=1}^{\infty} \sum_{m=0}^{n} \frac{1}{4 n(2 n-1) \lambda_{n m}}\left[4\left(1+\delta_{m 0}\right)(n-m)\right. \\
& \times(n-m-1)\left(\widetilde{P}_{n m}^{\alpha} \mathscr{D}_{n-1}^{m+1}-P_{n m}^{\alpha} \mathscr{D}_{n-1}^{m+1}\right)+\left(1-\delta_{m 0}\right) \\
& \left.\times(n+m)(n+m-1)\left(\widetilde{P}_{n m}^{\alpha} \mathscr{D}_{n-1}^{m-1}-P_{n m}^{\alpha} \mathscr{D}_{n-1}^{m-1}\right)\right]
\end{aligned}
$$

Finally, we determine the operator $\mathscr{H}^{\alpha}$ by comparing the terms proportional to $r^{-1-n} Y_{n}^{m}$ in the radial component of the singular part of the velocity, i.e., in

$$
\mathbf{r} \cdot \mathbf{u}^{\alpha, s}=\mathbf{r} \cdot\left[\mathscr{G}^{\alpha} r^{-1}-\nabla\left(\mathscr{G}^{\alpha} \cdot \nabla(r / 2)-\mathscr{H}^{\alpha} r^{-1}\right)\right] .
$$

From the definitions of $\mathscr{F}^{\alpha, T}$ and $\mathscr{G}^{\alpha, P}$, given respectively by (25)-(27) and (31)-(33), it can be shown that

$$
\begin{aligned}
\nabla \cdot \mathscr{G}^{\alpha}= & -\sum_{n=1}^{\infty} \sum_{m=0}^{n} \lambda_{n m}^{-1}\left[P_{n m}^{\alpha} \mathscr{D}_{n}^{m}+\widetilde{P}_{n m}^{\alpha} \mathscr{\mathscr { D }}_{n}^{m}\right. \\
& -\frac{\left(1+\delta_{m 0}\right)(n-m)(n-m-1)}{2 n(2 n-1)} \\
& \times\left(P_{n m}^{\alpha} \mathscr{D}_{n-2}^{m}+\widetilde{P}_{n m}^{\alpha} \widetilde{\mathscr{D}}_{n-2}^{m}\right) \nabla^{2}-\frac{1}{2}(n-m) \\
& \left.\times\left(\widetilde{T}_{n m}^{\alpha} \mathscr{D}_{n-1}^{m}-T_{n m}^{\alpha} \widetilde{\mathscr{D}}_{n-1}^{m}\right) \nabla^{2}\right] .
\end{aligned}
$$

Substituting the above expression for $\boldsymbol{\nabla} \cdot \mathscr{G}^{\alpha}$ into (34), using (14)-(18), and comparing the resulting expression with the singular part of the Lamb's solution (22), we obtain

$H_{n m}^{\alpha}=\lambda_{n m}^{-1}\left[\Phi_{n m}^{\alpha}-\frac{P_{n+2, m}^{\alpha}}{2(2 n+3)}\left(1-\frac{1+\delta_{m 0}}{n+2}\right)-\frac{1}{2} \widetilde{T}_{n+1, m}^{\alpha}\right]$,

$\widetilde{H}_{n m}^{\alpha}=\lambda_{n m}^{-1}\left[\widetilde{\Phi}_{n m}^{\alpha}-\frac{\widetilde{P}_{n+2, m}^{\alpha}}{2(2 n+3)}\left(1-\frac{1}{n+2}\right)+\frac{1}{2} T_{n+1, m}^{\alpha}\right]$.

This completes the determination of the differential operators in terms of the coefficients of the singular terms in the Lamb's solution [cf. (22)]. The next major task is to determine the relations between the coefficients that appear in the regular part of the Lamb's solution and those appearing in the general solution (20) with $\mathscr{G}$ and $\mathscr{H}$ defined above. The part of the velocity regular near $x^{\alpha}$ is

$$
\begin{aligned}
u_{i}^{\alpha, r} & =u_{i}-u_{i}^{\alpha, s} \\
& =\sum_{n=0}^{\infty}\left[\left(c_{n} r^{2} \nabla p_{n}^{\alpha}+b_{n} \mathbf{r} p_{n}^{\alpha}\right)+\nabla \times\left(\mathbf{r} \chi_{n}^{\alpha}\right)+\nabla \phi_{n}^{\alpha}\right] .
\end{aligned}
$$

The constants appearing in the above expression are related to certain derivatives of $u_{i}^{\alpha, r}$ evaluated at $\mathbf{x}^{\alpha}$. To determine the relation we use the method employed by Sangani and $\mathrm{Yao}^{17}$ which makes use of the following result for differentiation of spherical harmonics: ${ }^{15}$

$$
\begin{aligned}
\mathscr{D}_{k}^{l}\left(r^{n} Y_{n}^{m}\right)= & \frac{2^{-l}(n+m) !}{(n-k+m+l) !} r^{n-k} Y_{n-k}^{m+l} \\
& +\frac{(-2)^{-l}(n+m) !}{(n-k+|m-l|) !} r^{n-k} Y_{n-k}^{m-l},
\end{aligned}
$$

where, in evaluating the Legendre polynomials with negative order, we should use $P_{n}^{-m}=(-1)^{m} P_{n}^{m}$, and $P_{n}^{m}=0$ for $m>n$. Using this result it is easy to see that when (11) is operated by $\mathscr{D}_{k}^{l}$ and the result is evaluated at $r=0$ only the term with $P_{k l}^{\alpha, r}$ in it will survive. Thus, we have

$$
P_{k l}^{\alpha, r}=\eta^{-1} \epsilon_{k l} \mathscr{D}_{k}^{l} p^{\alpha, r}(0),
$$

where $p^{\alpha, r}$ is the regular part of pressure at $\mathbf{x}^{\alpha}$ and

$$
\epsilon_{k l}=\frac{(-2)^{l}}{\left(1+\delta_{l 0}\right)(k+l) !} .
$$

Thus, for example, substituting for the pressure from (28) into (40) we obtain

$$
P_{k l}^{\alpha, r}=\epsilon_{k l} \sum_{\gamma=1}^{N}\left[\mathscr{D}_{k}^{l}\left(\mathbf{x} \cdot \mathscr{G}^{\gamma} \nabla^{2}-\nabla \cdot \mathscr{G}^{\gamma}\right)\right] S_{1}\left(\mathbf{x}^{\alpha}-\mathbf{x}^{\gamma}\right) .
$$

Here, the derivatives of $S_{1}$ must first be evaluated with respect to $\mathrm{x}-\mathrm{x}^{\gamma}$ followed by the substitution $\mathrm{x}=\mathrm{x}^{\alpha}$. For $\alpha=\gamma$, the derivative of only the regular part of $S_{1}$, i.e., $S_{1}-1 / r$, at $r=0$ must be evaluated as the singular part has already been removed [cf. (38)]. In what follows, it will be understood that all the derivatives of $S_{1}$ and $S_{2}$ to be evaluated correspond to the regular parts of these functions whenever $\alpha$ and $\gamma$ are the same.

The expression for $\widetilde{P}_{k l}^{\alpha, r}$ is obtained by replacing $\mathscr{D}_{k}^{l}$ in (42) by $\mathscr{\mathscr { Q }}_{k}^{l}$.

The above idea can be extended to evaluate the regular parts of $\chi$ and $\phi$ also. Thus, we note that the scalar product $\mathbf{r} \cdot \omega^{\alpha, r}, \omega^{\alpha, r}$ being the regular part of the vorticity at $\mathbf{x}^{\alpha}$ [cf. (23)], satisfies the Laplace equation and, therefore, using (39) we obtain

$$
k(k+1) T_{k l}^{\alpha, r}=\left(\mathbf{r} \cdot \omega^{\alpha, r}\right)_{k l}=\epsilon_{k l} \mathscr{D}_{k}^{l}\left(\mathbf{r} \cdot \omega^{\alpha, r}\right)(0),
$$

where the subscript $k l$ in the second term stands for the coefficient of $r^{k} Y_{k}^{l}$ in the solid spherical harmonic expansion of that quantity. The first equality in the above expression is obtained from (38) by using the appropriate properties of poloidal fields (see Appendix III of Ref. 18 for details), while the second equality is obtained from the use of (39). Once again the expression for $\widetilde{T}_{k l}^{\alpha, r}$ is obtained by replacing $\mathscr{D}_{k}^{l}$ in (43) by $\widetilde{D}_{k}^{l}$.

To evaluate the derivatives required by (43), we make use of the following identity valid for any arbitrary function $\mathbf{f}$ obtained by using the simple chain rule of differentiation and the definitions (17) and (18):

$$
\begin{aligned}
\mathscr{D}_{k}^{l}(\mathbf{r} \cdot \mathbf{f})(0)= & (k-l) \mathscr{D}_{k-1}^{l} f_{1}(0) \\
& +\frac{l}{2}\left[\mathscr{D}_{k-1}^{l-1} f_{2}(0)-\widetilde{D}_{k-1}^{l-1} f_{3}(0)\right],
\end{aligned}
$$




$$
\begin{aligned}
\mathscr{D}_{k}^{l}(\mathbf{r} \cdot \mathbf{f})(0)= & (k-l) \mathscr{D}_{k-1}^{l} f_{1}(0) \\
& +\frac{l}{2}\left[\mathscr{D}_{k-1}^{l-1} f_{2}(0)+\mathscr{D}_{k-1}^{l-1} f_{3}(0)\right] .
\end{aligned}
$$

Now the Cartesian components of the vorticity can be evaluated with the use of (23), and substituting them into above expressions, we obtain formulas for evaluating $T_{k l}^{\alpha, r}$ and $\widetilde{T}_{k l}^{\alpha, r}$ as presented in Appendix $\mathrm{A}$.

We follow the same procedure for evaluating the regular components of $\phi_{n}$. These are related to the coefficients of solid spherical harmonics in $\psi \equiv \mathbf{r} \cdot \mathbf{u}^{\alpha, r}$. Since $\psi$ is biharmonic and regular at origin, we write its expansion in the Legendre polynomials as

$$
\psi=\sum_{k=0}^{\infty} \sum_{l=0}^{k}\left[\left(g_{k l}+r^{2} h_{k l}\right) r^{k} Y_{k}^{l}+\left(\widetilde{g}_{k l}+r^{2} \widetilde{h}_{k l}\right) r^{k} \widetilde{Y}_{k}^{l}\right]
$$

By comparing the above expression with the Lamb's solution, we see that $k \Phi_{k l}^{\alpha, r}=g_{k l}$ and $k \widetilde{\Phi}_{k l}^{\alpha, r}=\widetilde{g}_{k l}$. Taking Laplacian of (46) and using (39) we first obtain an expression for $h_{k l}$. Upon substituting for $h_{k l}$ into (46) and using (39) and similar formulas for differentiating $r^{2} Y_{n}^{m}$ from Ref. 16, we then obtain

$$
\begin{aligned}
h_{k l} & =\frac{\epsilon_{k l}}{4 k+6} \mathscr{D}_{k}^{l} \nabla^{2} \psi(0), g_{k l} \\
& =\epsilon_{k l}\left[\mathscr{D}_{k}^{l}-C \frac{(k-l)(k-l-1)}{4 k-2} \mathscr{D}_{k-2}^{l} \nabla^{2}\right] \psi(0),
\end{aligned}
$$

where $C$ equals zero for $k-l<2$ and unity otherwise. Once again the expression for $\widetilde{g}_{k l}$ and $\widetilde{h}_{k l}$ are obtained by replacing $\mathscr{\mathscr { V }}$ in $(47)$ by $\widetilde{D}$.

Detailed expressions for evaluating the regular parts of $\phi$ are also given in Appendix $\mathbf{A}$.

\section{BOUNDARY CONDITIONS}

One of the aims of the present study was to write a general purpose computer program for determining Stokes flow interactions in a variety of problems differing only through the boundary conditions at spherical surfaces. The formulation of the general solution in terms of constants appearing in the Lamb's solution is particularly suitable for treating various boundary conditions at the surface of drops, bubbles, charged particles, etc. The general methodology is to write the suitable expression for the velocity field inside the particles in a series of Legendre polynomials and then to combine it with the Lamb's solution for velocity outside the particle to obtain a set of equations satisfied by the regular and singular coefficients of the Lamb's solution. The result can then be recast into the following generic form in most cases:

$\Phi_{n m}^{\alpha, r}+\frac{(n+1) a^{-2 n+1}}{n(2 n-1)(2 n+1)} f_{n}^{s} P_{n m}^{\alpha}+\frac{a^{2}}{2(2 n+1)} f_{n}^{r} P_{n m}^{\alpha, r}=P_{n m}^{\alpha, \infty}$,

$$
\begin{aligned}
\Phi_{n m}^{\alpha} & -\frac{a^{2}}{2(2 n+1)} g_{n}^{s} P_{n m}^{\alpha}+\frac{n a^{2 n+3}}{(n+1)(2 n+1)(2 n+3)} g_{n}^{r} P_{n m}^{\alpha, r} \\
& =\Phi_{n m}^{\alpha, \infty},
\end{aligned}
$$

$T_{n m}^{\alpha, r}+a^{-2 n-1} h_{n}^{s} T_{n m}^{\alpha}=T_{n m}^{\alpha, \infty}$,

where $a$ is the radius of the particle $\alpha$ and $f, g$, and $h$ 's the coefficients that depend on the boundary conditions. For rigid particles with no-slip boundary condition, these coefficients are unity and (48)-(50) reduce to the expressions given in standard texts (e.g., Ref. 14). The constants with the superscript $\infty$ appearing on the right-hand side of the above equations depend on the translational and rotational velocities of the particle $\alpha$. In problems involving charged particles surrounded by a thin, polarized double layer, or drops moving under thermocapillary effect at low Marangoni numbers, the above constants also depend on the solution of electric potential or temperature around the particle. ${ }^{19}$ We hope to carry out dynamic simulations of some of these systems in our future work; in what follows we shall illustrate the application of the method by determining the transport coefficients of suspensions of drops and porous particles.

\section{A. Porous particles}

Porous particles are used as a model of macromolecules, catalyst pellets in fixed bed reactors, and supports used in immobilizing proteins in bioreactors. The question of determining the proper equations governing the flow inside the porous particles and the boundary conditions at the interface between the clear fluid and the porous particle has been addressed by a number of investigators. ${ }^{20-23} \mathrm{~A}$ review of this may be found in Refs. 22 and 23. We shall assume that the pore size $b$ characterizing the porous medium is small compared to the radius $a$ of the particle. Then, on the length scale of $a$, the flow inside the particles is generally well described by the Brinkman equation:

$$
\frac{1}{\hat{\eta}} \partial_{j} \sigma_{i j}=\nabla^{2} \mathbf{u}-\frac{1}{\hat{\eta}} \nabla p=\beta^{\prime 2}\left(\mathbf{u}-\mathbf{v}^{\alpha}\right), \quad \boldsymbol{\nabla} \cdot \mathbf{u}=0
$$

where $\beta^{\prime 2}$ is the inverse of Darcy permeability of the porous medium, $u$ the pore-ensemble-averaged velocity in the medium, and $\mathbf{v}^{\alpha}$ the velocity of the rigid skeleton of the particle given by

$$
\mathbf{v}^{\alpha}=\mathbf{U}^{\alpha}+\mathbf{\Omega}^{\alpha} \times_{\mathbf{r}}
$$

$\mathbf{U}^{\alpha}$ and $\boldsymbol{\Omega}^{\alpha}$ being, respectively, the translational and rotational velocities. We shall treat $\beta \equiv \beta^{\prime} a$ as an $O(1)$ quantity in the present study. This and the condition $b / a<1$ are satisfied for particles whose porosity is close to unity. The effective viscosity $\hat{\eta}$ of the porous medium can then be taken to be the same as that of the fluid, i.e., $\hat{\eta}=\eta$ (see, e.g. Ref. 24). (51) is

The solution for the flow inside the particle satisfying 


$$
\begin{aligned}
\mathbf{u}(\mathbf{r})= & \mathbf{v}^{\alpha}(\mathbf{r})+\sum_{n=1}^{\infty}\left[-\frac{1}{\beta^{\prime 2}} \nabla p_{n}^{\alpha}+\nabla \times\left(\mathbf{r} \chi_{n}\right)\right. \\
& \left.+\nabla \times \nabla \times\left(\mathbf{r} \phi_{n}\right)\right]
\end{aligned}
$$

where $p_{n}^{\alpha}$ is related to the pressure by $p=\eta \Sigma p_{n}^{\alpha}$, and

$$
\nabla^{2} p_{n}^{\alpha}=0, \quad \nabla^{2} \chi_{n}^{\alpha}=\beta^{\prime} \chi_{n}^{\alpha}, \quad \nabla^{2} \phi_{n}^{\alpha}=\beta^{\prime} \phi_{n}^{\alpha} .
$$

The boundary conditions at $r=a$ will be taken to be the continuity of velocity and traction. In the present case, i.e., the solenoidal velocity fields, the Newtonian stress tensors with equal viscosities, and the spherical geometry of the boundary, these boundary conditions can be shown to be equivalent to the continuity of $u_{r}, \partial_{r} u_{r}, \partial_{r}^{2} u_{r}, \omega_{r}, \partial_{r} \omega_{r}$, and $p$. Here, $\omega_{r}$ is the radial component of the vorticity. Now expressing the solution of (54) for $p$ in terms of the regular spherical harmonics and that for $\chi$ and $\phi$ in the modified spherical Bessel functions and applying the boundary conditions yield (48)-(50) with

$$
\begin{aligned}
& f_{n}^{s}=1+\frac{2 n-1}{\beta G_{n}(\beta)}+\frac{n(2 n-1)(2 n+1)}{(n+1) \beta^{2}} \\
& f_{n}^{r}=g_{n}^{s}=1-\frac{2}{G_{n}(\beta)}+\frac{2(2 n+1)}{\beta^{2}} \\
& g_{n}^{r}=1-\frac{2 n+3}{\beta G_{n}(\beta)}+\frac{(2 n+1)(2 n+3)}{\beta^{2}} \\
& h_{n}^{s}=\left[1-(2 n+1) G_{n}(\beta) \beta^{-1}\right]^{-1}
\end{aligned}
$$

Here, $G_{n}(\beta) \equiv i_{n}(\beta) / i_{n-1}(\beta), i_{n}(\beta)=(\pi / 2 \beta)^{1 / 2} I_{n+1 / 2}(\beta)$ being the modified spherical Bessel function (p. 443, Ref. 25 ). In the limit $\beta \rightarrow \infty$, i.e., vanishingly small permeability, all of the above coefficients approach unity and the case of rigid particles is recovered.

\section{B. Drops and bubbles}

For fluid particles with a viscosity $\hat{\eta}$ and a uniform surface tension, the usual boundary conditions are the continuity of the velocity and tangential stress together with a jump in the normal stress specified in terms of the interfacial tension and the local curvature. We shall consider only the limiting case of large interfacial tension for which the deformation of the particle is negligible. In this limiting case, we must discard the normal stress difference boundary condition and instead require that the normal component of the fluid motion at the boundary be the same as the projection of translational velocity of the drop along the normal vector. These conditions subsequently lead to (48)-(50) with the following definitions of $f, g$, and $h$ :

$$
\begin{aligned}
& f_{n}^{s}=\frac{1+\beta^{-1}}{1+2 \beta^{-1} /(2 n+1)}, \\
& f_{n}^{r}=g_{n}^{s}=1+\frac{2}{\beta(2 n+1)}, \\
& g_{n}^{r}=\frac{1-\beta^{-1}}{1+2 \beta^{-1} /(2 n+1)},
\end{aligned}
$$

$$
h_{n}^{s}=1+\frac{2 n+1}{(\beta-1)(n-1)} .
$$

Here, $\beta=\hat{\eta} / \eta$ is the viscosity ratio of the two fluids. Once again, all of the above constants approach unity as $\beta \rightarrow \infty$, and the case of rigid particles is recovered except for the $n=1$ equations in (50). The motion of rigid particles is specified by its translational and rotational velocities whereas that of drops and bubbles by the translational velocity alone. Consequently, it can be shown that the equation with $n=1$ in (50) can be discarded from the entire set of equations. For rigid particles, this equation is important only in determining the rotational velocity of the particles when the torque applied to the particle is specified.

\section{EXPRESSIONS FOR FORCE, TORQUE, STRESSLET, AND AVERAGE VELOCITY}

The average properties of the flow are related to $\mathbf{u}^{\infty}(\mathbf{x})$. To compute the average velocity, we simply integrate the velocity at all points in the unit cell and divide by the volume of the cell. Thus, we have

$$
\tau<u_{i}>=\int_{\tau} u_{i} d V+\sum_{\alpha=1}^{N} \int_{\gamma^{\alpha}}\left(u_{i}^{p, \alpha}-u_{i}\right) d V
$$

where $\tau$ is the volume of the cell, $V^{\alpha}$ the volume occupied by particle $\alpha$, and $u_{i}^{p, \alpha}$ the velocity inside particle $\alpha$. The velocity $u_{i}$ inside the particle will be defined to be the analytic extension of the velocity field outside the particle as defined by (5) or (20). Note that this quantity is singular at the particle centers and must, therefore, be treated as a generalized function in performing the integration. Also, it should be noted that in the case of porous particles $u_{i}^{p, \alpha}$ is the pore-averaged velocity of the fluid and not the velocity of the rigid skeleton.

For the purpose of evaluating various averaged quantities it is more convenient to use the expression (5) for u. Once the expressions for the quantities of interest are determined in terms of the constants appearing in (5) and (6), we relate them to the quantities such as $P_{n m}^{\alpha}$ that appear in the formal solution (20). Now when $u_{i}$ and $u_{i}^{p, \alpha}$ are both solenoidal, we can use the identity $\partial_{j}\left(\left(u_{j}-u_{j}^{p, \alpha}\right) r_{i}\right)=u_{i}-u_{i}^{p, \alpha}$ and the divergence theorem to convert the volume integral in the second term on the right-hand side to a surface integral. This integral therefore vanishes for all the problems in which the normal component of the velocity is continuous across the surface of the particle, and we thereby obtain $\left\langle u_{i}\right\rangle=U_{i}^{\infty}$. Note that in obtaining this result we have used the fact that while $u_{i}$ is itself singular at the center of the particle, its divergence is not. This is not true when there is a net source of fluid inside the particle, e.g. in applications involving a phase change at $r=a$. The above result, i.e., $\left\langle u_{i}\right\rangle=U_{i}^{\infty}$, however, holds even in such situations since the contribution from the singularity in the divergence of $u_{i}$ at $r=0$ is then balanced by the jump in the normal velocity at $r=a$.

The force on each particle may be evaluated once again by using the analytic extension of the stress in the fluid to that inside the particles. Thus, we have 


$$
F_{i}^{\alpha}=\int_{S^{\alpha}} \sigma_{i j} n_{j} d A=\int_{V^{\alpha}} \partial_{j} \sigma_{i j} d V=-4 \pi \eta A_{i}^{\alpha} .
$$

Here, $S^{\alpha}$ is the surface enclosing particle $\alpha$ and $n_{j}$ the unit normal outward vector on this surface. The last equality in (64) was obtained by making use of (1) and (6).

To compute the stresslet and torque, we use

$$
\begin{aligned}
\Lambda_{i j}^{\alpha} & \equiv \int_{S^{\alpha}} x_{i} \sigma_{j k} n_{k} d A \\
& =\int_{V^{\alpha}}\left(\sigma_{j i}+x_{i} \partial_{k} \sigma_{j k}\right) d V=\int_{V^{\alpha}} \sigma_{i j} d V+4 \pi \eta B_{i j}^{\alpha},
\end{aligned}
$$

where, in obtaining the last equality, we have made use of the results on integrals of generalized functions [note that from (1), (5), and (6), $\left.\partial_{k} \sigma_{j k}=-4 \pi \eta \mathscr{M}_{j}^{\alpha} \delta\left(\mathbf{x}-\mathbf{x}^{\alpha}\right)\right]$. The volume integral term on the extreme right side of (65) does not contribute to the stresslet or torque, which are given by

$$
\begin{aligned}
& S_{i j}^{\alpha} \equiv \frac{1}{2}\left(\Lambda_{i j}^{\alpha}+\Lambda_{j l}^{\alpha}-\frac{2}{3} \delta_{i j} \Lambda_{k k}^{\alpha}\right)-\eta \\
& \times \int_{S^{\alpha}}\left(u_{i} n_{j}+u_{j} n_{i}-\frac{2}{3} \delta_{i j} u_{k} n_{k}\right) d A \\
&= 2 \pi \eta\left(B_{i j}^{\alpha}+B_{j i}^{\alpha}\right), \\
& \mathscr{L}_{i}^{\alpha} \equiv \epsilon_{i j k} \Lambda_{j k}^{\alpha}=4 \pi \eta \epsilon_{i j k} B_{j k}^{\alpha} .
\end{aligned}
$$

The above results can now be rewritten in terms of $P_{n m}^{\alpha}$, etc. The difference between $u_{i}^{\infty}(\mathbf{x})$ and $U_{i}^{\infty}(\mathbf{x})$ arises because the term with the operator $\mathscr{H}$ in (20) is not entirely derived from the Laplacian of $v_{i j}$ in (5). It may be recalled that we chose $\mathscr{H}=-\partial_{j} \mathscr{M}_{j}^{L} \nabla^{2}$ plus a constant. Thus, we have

$$
\begin{aligned}
\mathscr{H}^{\alpha} \partial_{i} S_{1}= & \left(2 H_{10}^{\alpha} \delta_{j 1}+H_{11}^{\alpha} \delta_{j 2}+\tilde{H}_{11}^{\alpha} \delta_{j 3}\right) \partial_{i j} S_{1}+\cdots \\
= & \left(2 H_{10}^{\alpha} \delta_{j 1}+H_{11}^{\alpha} \delta_{j 2}+\tilde{H}_{11}^{\alpha} \delta_{j 3}\right)\left(4 \pi / \tau \delta_{i j}-\nabla^{2} v_{i j}\right) \\
& +\cdots,
\end{aligned}
$$

with the dots representing the higher-order derivatives of $S_{1}$. In deriving the above expressions we have made use of (19) and the relations $\mathscr{D}_{1}^{0}=2 \partial_{1}, \mathscr{D}_{1}^{1}=\partial_{2}$, and $\mathscr{D}_{1}^{1}=\partial_{3}$. Combining (68) with (5) and (20), and using (63), we obtain

$$
\left\langle u_{i}\right\rangle=U_{i}^{\infty}=u_{i}^{\infty}+\frac{4 \pi}{\tau} \sum_{\alpha=1}^{N}\left(2 H_{10}^{\alpha} \delta_{i 1}+H_{11}^{\alpha} \delta_{i 2}+\widetilde{H}_{11}^{\alpha} \delta_{i 3}\right) .
$$

In a similar manner, we can identify the first few terms in (20) with those in (6) to obtain

$$
\begin{aligned}
& F_{1}^{\alpha}=-4 \pi \eta P_{10}^{\alpha} \quad F_{2}^{\alpha}=4 \pi \eta P_{11}^{\alpha} \quad F_{3}^{\alpha}=4 \pi \eta \widetilde{P}_{11}, \\
& \mathscr{L}_{1}^{\alpha}=-8 \pi \eta T_{10}^{\alpha} \quad \mathscr{L}_{2}^{\alpha}=8 \pi \eta T_{11}^{\alpha} \quad \mathscr{L}_{3}^{\alpha}=8 \pi \eta \widetilde{T}_{11}^{\alpha},
\end{aligned}
$$

$$
\begin{aligned}
& S_{11}^{\alpha}=-\frac{4 \pi \eta}{3} P_{20}^{\alpha}, \quad S_{12}^{\alpha}=2 \pi \eta P_{21}^{\alpha}, \quad S_{13}^{\alpha}=2 \pi \eta \widetilde{P}_{21}^{\alpha}, \\
& S_{22}^{\alpha}=4 \pi \eta\left(P_{20}^{\alpha} / 6-P_{22}^{\alpha}\right), \quad S_{23}^{\alpha}=-4 \pi \eta \widetilde{P}_{22}^{\alpha}, \\
& S_{33}^{\alpha}=4 \pi \eta\left(P_{20}^{\alpha} / 6+P_{22}^{\alpha}\right) .
\end{aligned}
$$

The above expressions can alternatively be derived from the form of Lamb's solution around each sphere with the formulas given by Kim and Karrila (Sec. 4.2.2 in Ref. 26).

The mean pressure gradient in the medium is related to the first term on the right-hand side of (28) and is given by

$\partial_{i}\langle p\rangle=\frac{4 \pi \eta}{\tau} \sum_{\alpha=1}^{N}\left(P_{10}^{\alpha} \delta_{i 1}-P_{11}^{\alpha} \delta_{i 2}-\widetilde{P}_{11}^{\alpha} \delta_{i 3}\right)=-\frac{1}{\tau} \sum_{\alpha=1}^{N} F_{i}^{\alpha}$.

For fixed beds of rigid or porous particles a quantity of interest is the overall permeability which relates the mean flow to the mean pressure gradient. For isotropic beds, this is characterized by a scalar $k$ defined via

$$
\eta\langle\mathbf{u}\rangle=-k \nabla\langle p\rangle .
$$

Defining the nondimensional drag $F$ by $\left\langle F_{i}\right\rangle$ $=6 \pi \eta a\left\langle u_{i}\right\rangle F$, the overall permeability is related to the average drag force by

$$
k=\frac{9 a^{2}}{2 c F},
$$

where $c$ is the volume fraction of the particles.

In fixed beds of porous particles another quantity that is important is the magnitude of the average flow inside the particles. Integrating (51) over the volume of the particle $\alpha$, applying the divergence theorem, and using the fact that the traction is continuous at $r=a$, we find that

$$
\left\langle u_{i}^{\alpha}-v_{i}^{\alpha}\right\rangle^{p}=\frac{3\left\langle F_{i}\right\rangle}{4 \pi \eta a \beta^{2}}=\frac{9 F\left\langle u_{i}\right\rangle}{2 \beta^{2}},
$$

where the superscript $p$ above the averaging brackets refers to the average over the particle phase. Thus, the calculation of the average force, or equivalently, the pressure drop in the bed of porous particles also yields the estimate of the fluid velocity inside the porous particles.

The effective equations for the suspensions of porous particles can be derived using the method of ensemble averages. Thus, writing $\partial_{j} \sigma_{i j}+f_{i}=0$, with the force density $f_{i}$ equal to $\rho g_{i}$ for a point lying outside the porous particles and $\eta \beta^{\prime 2}\left(u_{i}-v_{i}\right)$ inside the particles, the ensembleaveraged momentum equation for the suspension is

$$
\partial_{j}\left\langle\sigma_{i j}\right\rangle+(1-c) \rho g_{i}+\left\langle f_{i}\right\rangle^{p}=0 .
$$

Here, $\rho$ is the density of the liquid and $g_{i}$ the gravitational acceleration. Note that the gravitational force inside the porous particles does not appear explicitly in the above equation. The quantity $\left\langle f_{i}\right\rangle^{p}$ is given by 


$$
\begin{aligned}
\left\langle f_{i}\right\rangle^{p}(\mathbf{x})= & \int_{s<a} f_{i}(\mathbf{s}, \mathbf{x}-\mathbf{s}) n(\mathbf{x}-\mathbf{s}) d V_{s} \\
= & n(\mathbf{x}) \int_{s<a} f_{i}(\mathbf{s}, \mathbf{x}) d V_{s} \\
& -\frac{\partial}{\partial x_{j}}\left\{n(\mathbf{x}) \int_{s<a} s_{j} f_{i}(\mathbf{s}, \mathbf{x}) d V_{s}\right\}+\cdots,
\end{aligned}
$$

where $f_{i}(\mathbf{s}, \mathbf{x})$ is the conditionally averaged force density at $\mathbf{x}+\mathbf{s}$ with a particle fixed at $\mathbf{x}$, and $n(\mathbf{x})$ the number density of the particles at $x$. In writing the second equality, we have made use of the Taylor series expansion of the integrand around $\mathbf{x}$. When the macroscopic properties vary slowly, the remaining terms in the expansion represented by the dots can be neglected and the averaged momentum equation (77) reduces to

$$
\partial_{j} \Sigma_{i j}+(1-c) \rho g_{i}-c\left\langle F_{i}\right\rangle=0,
$$

where $\left\langle F_{i}\right\rangle$ is the average hydrodynamic force on the particle and $\Sigma_{i j}$ the effective stress in the medium given by

$$
\Sigma_{i j}=\left\langle\sigma_{i j}\right\rangle-n \int_{s<a} s_{j} f_{i} d V_{s} .
$$

Note that the effective stress in this problem differs from the volume-averaged stress $\left\langle\sigma_{i j}\right\rangle$. It is now easy to show that the deviatoric part of the last term in the above equation is related to the average stresslet so that the effective stress can be represented for isotropic suspensions by means of a scalar effective viscosity

$$
\eta^{*}=\eta(1+n S)
$$

with $\left\langle S_{i j}\right\rangle=2 S e_{i j}, e_{i j}$ being the rate of strain tensor. Finally, by considering separately the momentum equation for the rigid skeleton of the particle, it can be shown that the sum of $\left\langle F_{i}\right\rangle$ and the gravity force $\left(\rho_{p}-\rho\right) g_{i} V_{p}$ must equal zero, so that the last two terms on the left-hand side of (79) can be combined to $\langle\rho\rangle g_{i}$. Here, $\rho_{p}$ is the density of the fiuid-saturated porous particles, $V_{p}$ the volume of the particle, and $\langle\rho\rangle=\rho+\left(\rho_{p}-\rho\right) c$ the average density of the suspension.

\section{MISCELLANEOUS DETAILS}

Equations (48)-(50) represent a set of linear equations in unknowns $T_{n m}^{\alpha}, P_{n m}^{\alpha}, \Phi_{n m}^{\alpha}$ and the corresponding quantities with tilde. Note that the regular coefficients $T_{n m}^{\alpha, r}$, etc., are related to the above via the relations given in Appendix A. This infinite set of equations is first truncated to a set of finite equations, and the resulting equations are solved to determine the coefficients and hence the various transport properties. The truncation is based on an analysis of the equations for the special case of well-separated arrays for which the distance between any two particles is much greater than the radius of the particles. This analysis suggests that (48) should be truncated to $n<N_{s}$, (49) to $N_{s}-2$, and (50) to $N_{s}-1$. The unknowns $P_{n m}$ (or $\widetilde{P}_{n m}$ ) should likewise be truncated to $N_{s}, T_{n m}$ to $N_{s}-1$, and $\Phi_{n m}$ to $N_{s}-2$. This results in a total of $\left(3 N_{s}^{2}-1\right) N$ un- knowns for $N_{s} \geqslant 2$, and $3 N$ for $N_{s}=1$. The basis for such a truncation can be understood from the details of the analyses of dilute periodic arrays (Refs. 12 and 16). For concentrated arrays, of course, there is no guarantee that such a truncation scheme will always produce successively better estimates as $N_{s}$ is increased, but the experience with a number of calculations of this nature has shown the scheme to be successful in most cases. In computations, we make one further modification to $(48)-(50)$ and replace the unknown $\Phi_{n m}^{\alpha}$ by $a^{2} g_{n}^{s} P_{n m}^{\alpha} /(2(2 n+1))+\Phi_{n m}^{*} \alpha$. This improved the convergence properties of the numerical scheme. It may be noted that, with $g_{1}^{s}=1$, this is equivalent to replacing the leading Stokeslet term $A_{j} v_{i j}$ in the formal solution by $A_{j}\left(1+\left(a^{2} / 6\right) \nabla^{2}\right) v_{i j}$ corresponding to the flow past an isolated sphere of radius $a$, or, equivalently, replacing the point force at center with a uniform distribution of force density at the surface of the sphere.

The computer program for Stokes flow interactions consists of three parts. The first part is concerned with the evaluation of derivatives $\mathscr{D}_{n}^{m} S_{1}\left(\mathbf{x}^{\alpha}-\mathbf{x}^{\gamma}\right)$, etc. There are a total of $N(N-1) / 2$ pairs in the suspension, and for each pair, the derivatives must be evaluated for $n \leqslant 2 N_{s}$. These derivatives are evaluated using the Ewald summation technique; the formulas for which may be found in Sangani et $a .^{27}$ Only the expressions for derivatives of $S_{1}$ were given in that reference but those of $S_{2}$ can be evaluated in a similar manner. For each pair, the total number of derivatives to be evaluated equals $\left(2 N_{s}+1\right)\left(2 N_{s}+2\right)$. At present, this is computationally the most intensive step. The second and third parts of the program consist of setting up the coefficients of a matrix whose size is $N_{t}=\left(3 N_{s}^{2}-1\right) N$ and solving the resulting set of linear equations. The time for setting up is negligible while that for solving is typically no more than $20 \%$ of the first part. The overall computations roughly scale as $N^{2} N_{s}^{2}$.

\section{RESULTS}

We shall present results for permeability, sedimentation velocity, and effective viscosity of suspensions of drops and porous particles. The hard-sphere random distributions were generated using a molecular dynamics code. The results for random arrays were obtained by averaging over 20 configurations with the same volume fraction $c$ of particles, except for few cases for which we carried out calculations with 100 configurations to compare our results with that of Ladd ${ }^{11}$ who obtained the corresponding results for rigid particles. Since the hard-sphere distribution undergoes a phase transition at higher $c$, the calculations for these random distributions were limited to $c \leqslant 0.45$. In many fixed bed operations, $c$ of interest is closer to 0.6 , and therefore additional calculations were made for randomly packed stable arrays of spheres. The computer program for generating such stable arrays developed recently by Professor Goddard's research group was used for this purpose. The details of the algorithm may be found in Ref. 28. Once again, the results for the packed arrays were obtained by averaging over 20 independent configurations with $c=0.611$. 
TABLE I. Convergence of numerical results for the viscous drag ratio $F / F_{0}$ with $N_{s}$.

\begin{tabular}{ccccccccc}
\hline \hline $\mathrm{c}$ & $N_{s} \backslash \beta$ & $\infty$ & 50 & 20 & 10 & 5 & 1 & 0.1 \\
\hline \multirow{6}{*}{0.45} & 2 & 10.439 & - & 9.482 & 7.531 & 4.478 & 1.229 & 1.00236 \\
& 3 & 22.952 & - & 15.105 & 9.564 & 4.782 & 1.230 & 1.00236 \\
& 4 & 25.815 & - & 15.974 & 9.795 & 4.809 & 1.230 & \\
& 5 & 27.226 & - & 16.319 & 9.875 & 4.817 & & \\
& 6 & 27.850 & - & 16.430 & 9.896 & & & \\
& 7 & 28.099 & & & & & & \\
\hline \multirow{6}{*}{0.611} & 2 & 9.89 & 10.35 & 10.32 & 8.79 & 5.179 & 1.2479 & 1.002526 \\
& 3 & 52.23 & 43.25 & 28.33 & 14.45 & 5.859 & 1.2485 & 1.002526 \\
& 4 & 68.57 & 53.25 & 31.56 & 14.99 & 5.901 & 1.2485 & \\
& 5 & 82.02 & 59.98 & 33.27 & 15.23 & 5.919 & & \\
& 6 & 92.71 & 64.34 & 34.11 & 15.33 & 5.924 & & \\
& 7 & 100.27 & 66.85 & 34.48 & & & & \\
& 8 & 103.79 & 67.78 & & & & & \\
& 9 & 105.19 & 68.06 & & & & & \\
\hline \hline
\end{tabular}

\section{A. Porous particles}

Table I shows the convergence of the numerical results for the average drag force per particle in fixed beds of Brinkman particles. The force on an isolated Brinkman particle nondimensionalized with the Stokes drag $6 \pi \eta a U^{\infty}$ is given by

$$
F_{0}=\frac{1}{f_{1}^{s}}
$$

with $f_{1}^{s}$ given by (55). This force, which has been obtained previously by Debye and Bueche ${ }^{29}$ and Felderhof, ${ }^{30}$ is plotted in Fig. 1. Note that in the limit of small and large $\beta$, $F_{0}$ is given, respectively, by

$$
F_{0}=\frac{2}{9} \beta^{2}\left(1-\frac{4}{15} \beta^{2}+O\left(\beta^{4}\right)\right) \quad(\beta \rightarrow 0),
$$

and

$$
F_{0}=1-\beta^{-1}+O\left(\beta^{-2}\right) \quad(\beta \rightarrow \infty) .
$$

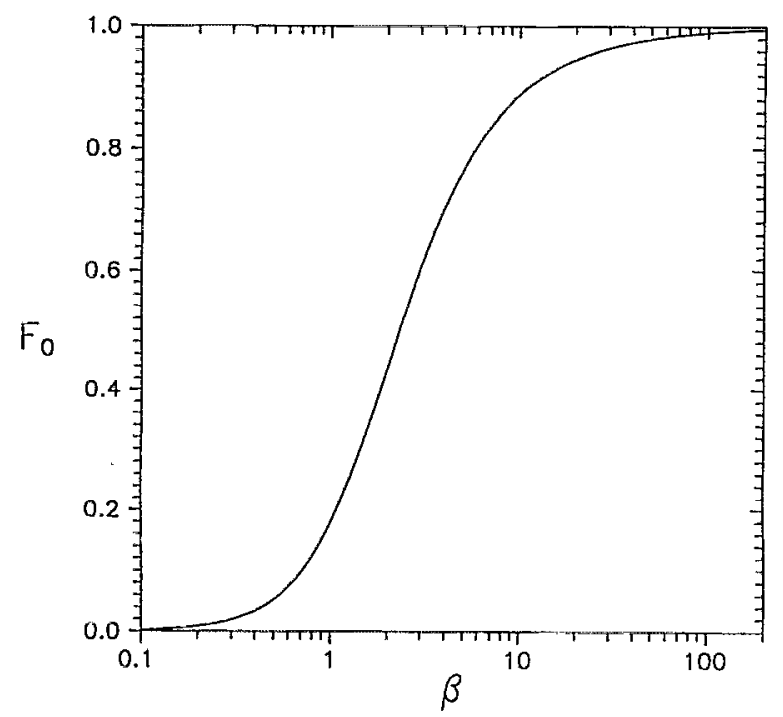

FIG. 1. The nondimensional drag force $F_{0}$ as a function of $\beta$.
TABLE II. $F / F_{0}$ as a function of $c$ and $\beta$ for the random arrays with $N=16$.

\begin{tabular}{lcccccc}
\hline \hline$c \backslash \beta$ & $\infty$ & 20 & 10 & 5 & 1 & 0.1 \\
\hline 0.1 & 2.8 & 2.6 & 2.34 & 1.95 & 1.115 & 1.00127 \\
0.25 & 7.1 & 5.7 & 4.52 & 3.05 & 1.177 & 1.00188 \\
0.35 & 14.2 & 10.0 & 7.01 & 3.98 & 1.210 & 1.00218 \\
0.45 & 28.1 & 16.5 & 9.9 & 4.82 & 1.230 & 1.00236 \\
0.611 & 107 & 34.5 & 15.4 & 5.93 & 1.249 & 1.00253 \\
\hline \hline
\end{tabular}

In Table I $N_{s}$ represents the order of force multipoles retained in determining the average property. Thus, for example, $N_{s}=6$ corresponds to keeping up to $2^{5}$ force multipoles in the formal solution for the velocity. As mentioned in the previous section, the total number of multipoles per particle varies as $3 N_{s}^{2}-1$. The number of particles $N$ per unit cell is 16 for the calculations presented in the table. Our results for rigid particles, $\beta=\infty$, are in very good agreement with those reported by Ladd ( $p_{\max }$ in his study equals our $N_{s}-1$ ). For c $=0.45$, his results with $N_{s}=7$ are about $1.5 \%$ greater. This slight difference resulted from using only 20 configurations to determine the averaged drag force; in fact, upon repeating the calculations with $\beta=\infty$ over 100 configurations we found the perfect agreement with his results. In view of the additional parameter $\beta$ in our problem, we continued to use only 20 configurations for averaging. It is interesting to note that the effect of $c$ is negligible for $\beta \leqslant 1$. The calculations for $c=0.611$ converge slowly, and since the number of simultaneous equations to be solved increase rapidly with $N_{s}$, we have carried out calculations only up to $N_{s}=9$. Calculations were made for a single configuration up to $N_{s}=11$, and it was found that the increase was about $2 \%$. Based on this calculation, and with the convergence tests on the body-centered cubic arrays with $N=1$ for which it is possible to carry out calculations to much higher $N_{s}$, we expect the result with $N_{s}=9$ to be lower and within about $5 \%$ of the actual result. The accuracy for smaller $\beta$ should, of course, be much better.

Table II gives the values of $F / F_{0}$ for several different $c$ and $\beta$. These results were obtained with $N=16$. The effect of finite $N$ on the average force calculations has been examined in detail by Ladd ${ }^{11}$ who found it to be generally small. For example, at $c=0.45$, the force ratio varied from 28.6 for $N=16$ to 28.2 for $N=108$, a difference of about $1.4 \%$. The velocity disturbance caused by a single particle is screened by the Brinkman term in the one-particle conditionally averaged equations, and this is mainly responsible for the small observed dependence on $N$.

The Carman-Kozeny ${ }^{31,32}$ relation

$$
\frac{F}{F_{0}}=\frac{10 c}{(1-c)^{3}} \quad(\beta=\infty),
$$

is known to predict the force on packed beds of monodispersed nonporous particles quite accurately. It is interesting to note that, with $c=0.611$, this relation gives $F / F_{0}=104$, in excellent agreement with our estimate of 107. 
TABLE III. $F / F_{0}$ for the simple cubic arrays of porous particles.

\begin{tabular}{lccccc}
\hline \hline$c \backslash \beta$ & $\infty$ & 50 & 20 & 10 & 1 \\
\hline 0.05 & 2.5070 & 2.4414 & 2.3404 & 2.1737 & 1.1217 \\
0.1 & 3.6424 & 3.4920 & 3.2636 & 2.9029 & 1.1531 \\
0.2 & 6.7759 & 6.2855 & 5.5575 & 4.5085 & 1.1896 \\
0.25 & 9.0623 & 8.2544 & 7.0673 & 5.4438 & 1.2020 \\
0.3 & 12.064 & 10.774 & 8.8977 & 6.4789 & 1.2120 \\
0.35 & 16.02 & 14.003 & 11.103 & 7.6091 & 1.2203 \\
0.4 & 21.24 & 18.13 & 13.728 & 8.8188 & 1.2272 \\
0.45 & 28.09 & 23.36 & 16.79 & 10.08 & 1.2330 \\
0.5 & 37.00 & 29.90 & 20.29 & 11.36 & 1.2378 \\
0.5236 & 42.1 & 33.5 & 22.08 & 11.96 & 1.2398 \\
\hline \hline
\end{tabular}

Since many practical applications of fixed beds involve high $c$ values, we give in Tables III-V the corresponding results for the three cubic arrays. It is possible to use $N_{s}=15$ since $N=1$ in these calculations, and thus the results presented in these tables are expected to be quite accurate. Comparing these tables, we see that the drag force on particles in a periodic array is generally greater than that for random arrays up to about $c=0.45$. At $c=0.611$, the estimate for random arrays is greater than that for the body-centered cubic array but less than that for the face-centered cubic array. The results for the rigid particles are in excellent agreement with those available in the literature. ${ }^{16,32}$

Results for porous particles were checked against three different asymptotes. The first two are based on the asymptotic analyses for large and small $\beta$ due to Kojima and Higdon. ${ }^{22}$ These asymptotes apply at arbitrary $c$. The third is the low $c$ asymptote for arbitrary $\beta$.

-The high and low $\beta$ asymptotes in Ref. 22 were determined for a single porous particle of arbitrary shape, but their analysis can be readily modified to the present problem. When the particles are only slightly porous, $\beta>1$, the use of reciprocal theorem together with the leading approximation for the velocity of the fluid at the particle surface obtained from the integral equation representation of Kojima and Higdon gives

$$
F(c, \beta)=F(c, \infty)-\lambda_{1} \beta^{-1},
$$

TABLE IV. $F / F_{0}$ for the body-centered cubic arrays of porous particles.

\begin{tabular}{lccccc}
\hline \hline$c \backslash \beta$ & $\infty$ & 50 & 20 & 10 & 1 \\
\hline 0.05 & 2.5697 & 2.5006 & 2.3944 & 2.2191 & 1.1243 \\
0.1 & 3.7656 & 3.6080 & 3.3692 & 2.9902 & 1.1565 \\
0.2 & 7.0416 & 6.5335 & 5.7847 & 4.6952 & 1.1942 \\
0.25 & 9.4441 & 8.6019 & 7.3808 & 5.6956 & 1.2070 \\
0.3 & 12.657 & 11.289 & 9.3425 & 6.8170 & 1.2174 \\
0.35 & 17.04 & 14.830 & 11.766 & 8.0672 & 1.2260 \\
0.4 & 23.11 & 19.55 & 14.760 & 9.4459 & 1.2332 \\
0.45 & 31.69 & 25.92 & 18.44 & 10.94 & 1.2392 \\
0.5 & 44.02 & 34.58 & 22.93 & 12.53 & 1.2443 \\
0.5236 & 51.7 & 39.7 & 25.35 & 13.30 & 1.2464 \\
0.6 & 88.9 & 62.5 & 34.52 & 15.81 & 1.2522 \\
0.611 & 96.4 & 66.8 & 36.01 & 16.17 & 1.2529 \\
0.65 & 129.1 & 84.1 & 41.50 & 17.39 & 1.2551 \\
0.6802 & 163 & 100.4 & 46.0 & 18.29 & 1.2566 \\
\hline \hline
\end{tabular}

TABLE V. $F / F_{0}$ and $\lambda_{1}$ for the face-centered cubic arrays of porous particles.

\begin{tabular}{lcccccc}
\hline \hline$c \backslash \beta$ & $\infty$ & 50 & 20 & 10 & 1 & $\lambda_{1}$ \\
\hline 0.05 & 2.5693 & 2.5002 & 2.3941 & 2.2189 & 1.1243 & 5.963 \\
0.1 & 3.7646 & 3.6070 & 3.3683 & 2.9896 & 1.1565 & 11.52 \\
0.2 & 7.0532 & 6.5409 & 5.7876 & 4.6951 & 1.1942 & 32.30 \\
0.25 & 9.4901 & 8.6327 & 7.3955 & 5.6986 & 1.2070 & 51.71 \\
0.3 & 12.793 & 11.379 & 9.3858 & 6.8274 & 1.2174 & 82.25 \\
0.35 & 17.38 & 15.054 & 11.869 & 8.0916 & 1.2260 & 131.3 \\
0.4 & 23.91 & 20.06 & 14.977 & 9.4933 & 1.2332 & 211.7 \\
0.45 & 33.49 & 27.00 & 18.86 & 11.02 & 1.2392 & 347.4 \\
0.5 & 47.97 & 36.77 & 23.67 & 12.66 & 1.2443 & 583.8 \\
0.5236 & 57.4 & 42.7 & 26.30 & 13.45 & 1.2464 & 753.4 \\
0.6 & 107.7 & 70.8 & 36.45 & 16.05 & 1.2522 & 1812 \\
0.611 & 118.8 & 76.3 & 38.12 & 16.42 & 1.2529 & 2070 \\
0.65 & 170.5 & 99.5 & 44.28 & 17.68 & 1.2551 & 3360 \\
0.6802 & 230 & 122 & 49.3 & 18.60 & 1.2566 & 4939 \\
0.7 & 281 & 139 & 52.6 & 19.17 & 1.2575 & 6370 \\
0.7405 & 432 & 180 & 59.2 & 20.23 & 1.2591 & 10778 \\
\hline \hline
\end{tabular}

where

$$
\lambda_{1}=\frac{1}{6 \pi U^{\infty 2}}\left\langle\int_{S^{\alpha}} \omega \cdot \omega d A\right\rangle,
$$

where $\omega$ is the vorticity, $F$ the force nondimensionalized by $6 \pi a \eta U^{\infty}$, and the angular brackets denote the average over all the particles. The vorticity can be related to the unknowns $P_{n m}$, etc., as shown in Appendix B so that it is possible to obtain an analytical expression for $\lambda_{1}$ in terms of the force multipoles. Numerical values of $\lambda_{1}$ for the face-centered cubic array are given in Table V. The convergence of this quantity with $N_{s}$ was found to be rather slow and therefore we did not determine $\lambda_{1}$ for random arrays.

For highly porous particles, Kojima and Higdon started with an integral equation representation for the velocity in terms of the Stokeslet distribution at the surface of particles and obtained a leading estimate for the force by substituting in the integral equation the approximately uniform velocity distribution. Replacing the Oseen tensor in their analysis for a single particle by $v_{i j}=S_{1} \delta_{i j}-\partial^{2}{ }_{i j} S_{2}$, the tensor for periodic lattice, we obtain for the dimensional force

$$
\begin{aligned}
F_{i}^{\alpha}= & \eta a^{-2} \beta^{2} V_{p} U_{i}^{\infty}-\frac{\eta \beta^{4} a^{-4}}{4 \pi} U_{j}^{\infty} \\
& \times \sum_{\gamma=1}^{N} \int_{V^{\alpha}} \int_{V^{\gamma}} v_{i j}(\mathbf{x}-\mathbf{y}) d V_{\mathbf{x}} d V_{\mathbf{y}},
\end{aligned}
$$

where $V_{p}=4 \pi a^{3} / 3$ is the volume of particles, and $\mathbf{x}$ and $\mathbf{y}$ are the dummy variables of integration inside the particles $\alpha$ and $\gamma$. Now using (83) for $F_{0}$, the above result can be recast to

$$
\frac{F}{F_{0}}=1-\lambda_{2} \beta^{2}+O\left(\beta^{4}\right) .
$$

Details of evaluating analytically the integrals in (88) and hence $\lambda_{2}$ are given in Appendix B. For the simple cubic array we obtain 


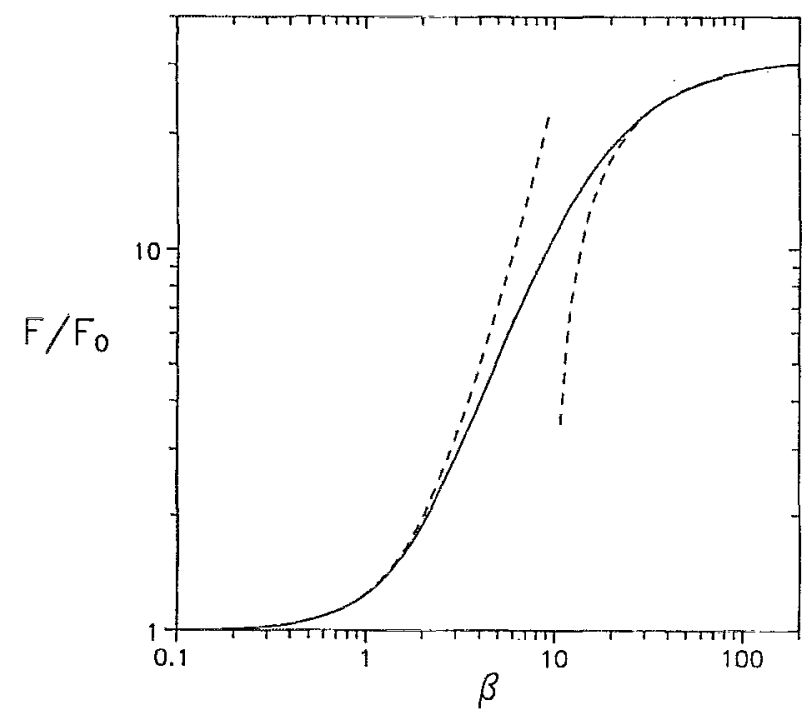

FIG. 2. $F / F_{0}$ versus $\beta$ for a body-centered cubic array with $c=0.45$. The solid line is the exact result and the dashed lines are the asymptotes for small and large $\beta$.

$$
\lambda_{2}=\frac{2}{9}\left[-1.7601 c^{1 / 3}+3 c / 5\right],
$$

valid for all $c$.

Figure 2 shows the exact numerical results for bodycentered cubic array with $c=0.45$ versus the two asymptotes. The large $\beta$ asymptote is seen to agree well with the exact results for $\beta>30$ and the small $\beta$ asymptote for $\beta<1$. Attempts at forming a composite expression based on the above two asymptotes that will essentially reproduce the entire exact results curve were not successful.

Finally, the analysis for small $c$ and arbitrary $\beta$ follows that of Hasimoto ${ }^{12}$ and Sangani and Acrivos. ${ }^{16}$ The final result for a simple cubic array is

$$
\begin{gathered}
\frac{F_{0}}{F}=1-\frac{1.7601}{f_{1}^{s}} c^{1 / 3}+\frac{f_{1}^{r}+g_{1}^{r}}{2 f_{1}^{s}} c-\left(\frac{g_{1}^{r}}{5 f_{1}^{s}}+\frac{1.3593}{f_{1}^{s} f_{3}^{s}}\right) c^{2} \\
+\frac{1.3930\left(f_{1}^{r}+g_{1}^{s}\right)+1.1940 f_{3}^{r}}{f_{1}^{s} f_{3}^{s}} c^{8 / 3}+\cdots,
\end{gathered}
$$

which for the case of rigid particles agrees with that given by Sangani and Acrivos. In the opposite limit of high permeability, the terms of $O\left(c^{2}\right)$ and $O\left(c^{8 / 3}\right)$ can be shown to be $O\left(\beta^{4}\right)$, and thus, the above expression is also consistent with the low $\beta$ asymptote (89). The above expression predicts accurate values of $F$ up to about $c=0.2$ for simple cubic arrays.

It is of some interest to compare the exact results with those predicted by the approximate theories such as the self-consistent field approximation and the cell theory. ${ }^{33,34}$ Recently, Davis and Stone ${ }^{34}$ obtained estimates for the average force in fixed beds of Brinkman as well as Darcy particles. In the self-consistent approximation, which the authors referred to as the swarm model, the force is determined by solving the flow around and through the Brinkman particle surrounded by a fluid cell up to a radius

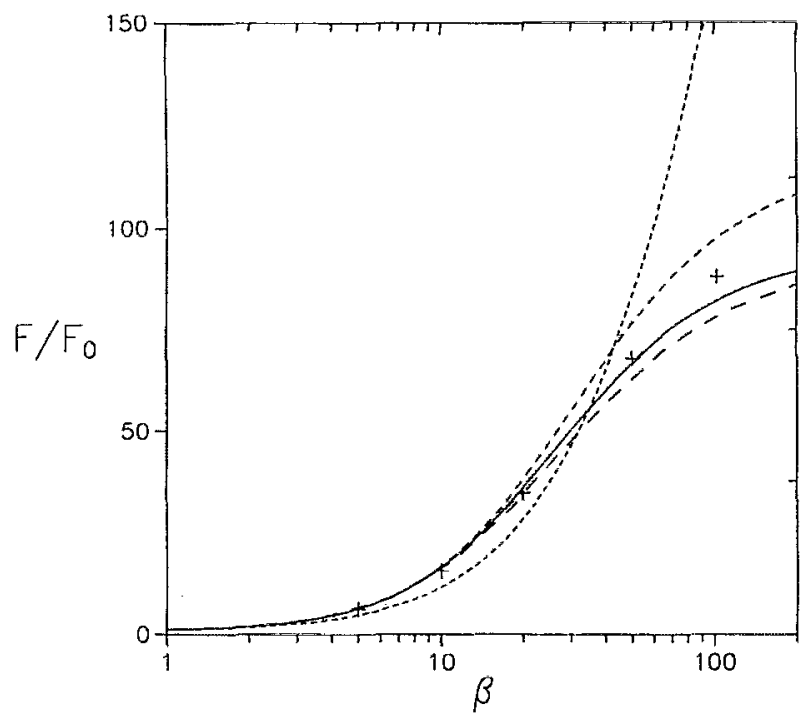

FIG. 3. $F / F_{0}$ versus $\beta$ for $c=0.611$. The solid line is the result for the body-centered cubic array, the medium dashed line for the face-centered cubic array, pluses for the random packed arrays, the small dashed line for the swarm theory, and large dashed line for the cell theory.

$a c^{-1 / 3}$, and an effective medium beyond this distance with a permeability that is related to the force on the particle. In the cell theory, the Brinkman medium beyond the radius $a c^{-1 / 3}$ is removed and instead the flow is required to offer a zero tangential stress at $r=a c^{-1 / 3}$. Further details and the final estimates may be found in their paper. Figure 3 shows a comparison between the exact results for the periodic as well as random arrays at $c=0.611$ determined in the present study with the estimates obtained from these two approximate theories. We find that the estimates from the cell theory are remarkably accurate.

We now turn to the problem of determining the effective viscosity $\eta^{*}$ of hard-sphere random distributions of porous particles. For dilute suspensions the effective viscosity is given by

$$
\eta^{*}=\eta\left[1+S(\beta) c+O\left(c^{2}\right)\right],
$$

with $S(\beta)=5 /\left(2 f_{2}^{s}\right)$. This estimate of the effective viscosity is in agreement with those given previously by Debye and Bueche ${ }^{29}$ and Felderhof. ${ }^{35}$ In the limit of large and small $\beta, S$ approaches $5 / 2$ and $\beta^{2} / 10-\beta^{4} / 175$, respectively.

Table VI shows the convergence of the effective viscosity with $N_{s}$ for $c=0.45$. The relative motion between particles gives rise to large lubrication forces in the case of rigid particles, and consequently, large $N_{s}$ will be required to evaluate accurately the effective viscosity. Nevertheless, our result with $N_{s}=10$ for rigid particles is only $1.8 \%$ lower than that obtained by Ladd who used the method of Brady and Bossis to account for the lubrication forces explicitly. We expect our results for other $\beta$ values to be even more accurate. The results for other values of $c$ are given in Table VII. These results were obtained with $N=16$. As shown by Ladd, the effect of $N$ on the effective viscosity of hard-sphere distributions is negligible. This can be ex- 
TABLE VI. The effective viscosity $\eta^{*} / \eta$ of random arrays of porous particles with $N=16$ and $c=0.45$ as a function of $N_{s}$.

\begin{tabular}{rcccccc}
\hline \hline$N_{s} \backslash \beta$ & $\infty$ & 20 & 10 & 5 & 1 & 0.1 \\
\hline 2 & 3.225 & 2.623 & 2.155 & 1.613 & 1.04335 & 1.0004498 \\
3 & 3.596 & 2.802 & 2.225 & 1.624 & 1.04335 & \\
4 & 4.165 & 3.004 & 2.285 & 1.631 & & \\
5 & 4.557 & 3.096 & 2.305 & 1.633 & & \\
6 & 4.832 & 3.135 & 2.311 & & & \\
7 & 5.040 & 3.151 & & & & \\
8 & 5.206 & & & & & \\
9 & 5.323 & & & & & \\
10 & 5.39 & & & & & \\
\hline
\end{tabular}

plained as follows. At small $c, \eta^{*}$ is given by (92) for both random as well as periodic arrays, and thus, the effect of finite $N$ is unimportant, At large $c$, the stresslet and hence $\eta^{*}$ is largely determined by the nearest neighbor distribution, which is generally well captured with a relatively small $N$. Indeed, Ladd's calculations with $N$ varying from 16 to 108 gave an increase of only $1.8 \%$ at $c=0.45$. The difference for smaller $c$ is even smaller, and, in some cases, negative.

As shown by Phillips et al. ${ }^{36}$ and Ladd, ${ }^{11}$ the finite $N$ effect is significant for the sedimentation problem due to the difference in the limiting behavior of dilute random and ordered suspensions and due to the lack of hydrodynamic screening in random sedimenting suspensions. To determine the finite $N$ correction, we divide the suspension into two regions: the inner region near the test particle which is adequately modeled in our computations, and the outer region of length scale $h$ in which the test particle has its periodic images. The apparent viscosity of the suspension on the outer region scale is the effective viscosity $\eta^{*}$. The flow induced due to periodicity in the outer region differs from that due to random suspensions because of the incorrect force density distribution near the periodic images of the test particle. ${ }^{37}$ More specifically, the difference in the velocity induced at the center of the test particle in a periodic suspension and the random suspension equals

$$
\sum_{\mathrm{x}_{\mathrm{L}}} \frac{F_{j}}{4 \pi \eta} \int d V_{\mathrm{y}}[P(\mathbf{y} \mid \mathbf{0})-P(\mathbf{y})] G_{i j}\left(\mathbf{y}+\mathrm{x}_{\mathrm{L}}\right) \quad\left(\mathrm{x}_{\mathrm{L}} \neq \mathbf{0}\right),
$$

where $\mathbf{y}$ is measured from $\mathbf{x}_{\mathbf{L}}, P(\mathbf{y})=n$ the number density of the particles, $P(\mathbf{y} \mid \mathbf{0})=n g(\mathbf{y} \mid \mathbf{0})$ the conditional probability density given a particle at $\mathbf{x}_{\mathbf{L}}, F_{j}$ the gravity force on the particles, and $G_{i j}=\left(\delta_{i j} / s+s_{i} s_{j} / s^{3}\right) / 2$ the Stokes propagator with $\mathbf{s}=\mathbf{y}+\mathbf{x}_{\mathbf{L}}$. When $h$ is much larger than the

TABLE VII. The effective viscosity $\eta^{*} / \eta$ of random arrays of porous particles.

\begin{tabular}{lcccccc}
\hline \hline$c \backslash \beta$ & $\infty$ & 20 & 10 & 5 & 1 & 0.1 \\
\hline 0.1 & 1.313 & 1.246 & 1.190 & 1.114 & 1.0095 & $1+1.0001686 \times 10^{-4}$ \\
0.25 & 2.201 & 1.813 & 1.573 & 1.313 & 1.0239 & 1.0002505 \\
0.35 & 3.139 & 2.333 & 1.890 & 1.461 & 1.0336 & 1.0003515 \\
0.45 & 5.4 & 3.16 & 2.31 & 1.633 & 1.0434 & 1.0004498 \\
\hline \hline
\end{tabular}

TABLE VIII. Sedimentation velocity $U / U_{0}$ for random suspensions of porous particles. The values enclosed within parentheses are the extrapolated values for $N=\infty$.

\begin{tabular}{cccccc}
\hline \hline$c$ & $N \backslash \beta$ & $\infty$ & 20 & 10 & 5 \\
\hline & 16 & $0.412(0.53)$ & $0.442(0.55)$ & $0.477(0.59)$ & $0.553(0.65)$ \\
& 32 & $0.438(0.53)$ & $0.468(0.56)$ & $0.502(0.59)$ & $0.576(0.66)$ \\
0.1 & 54 & $0.449(0.52)$ & $0.478(0.55)$ & $0.513(0.59)$ & $0.586(0.65)$ \\
& 108 & $0.460(0.52)$ & $0.490(0.55)$ & $0.524(0.58)$ & $0.596(0.65)$ \\
\hline 0.25 & 16 & $0.175(0.20)$ & $0.208(0.24)$ & $0.252(0.29)$ & $0.353(0.39)$ \\
\hline 0.35 & 16 & $0.086(0.096)$ & $0.116(0.129)$ & $0.158(0.173)$ & $0.264(0.281)$ \\
\hline 0.45 & 16 & $0.042(0.045)$ & $0.068(0.072)$ & $0.108(0.114)$ & $0.213(0.220)$ \\
\hline \hline
\end{tabular}

correlation length scale of the pair distribution function, we may expand $G_{i j}$ in a Taylor series around $\mathbf{x}_{\mathbf{L}}$ and determine the velocity correction using only the leading term. On making use of the result that the sum of $G_{i j}$ over all the periodic images in a simple cubic lattice equals $v_{i j}^{r}(0)$ or $-1.1734 a^{-1}(c / N)^{1 / 3} \delta_{i j}$, and $F=6 \pi a \eta U_{0} / f_{1}^{s}, U_{0}$ being the velocity of the isolated particle [cf. (55) and (82)], we find that the sedimentation velocity of the particle in the limit $N \rightarrow \infty$ is given by

$$
U(\infty)=U(N)+\frac{1.7601 \eta}{f_{1}^{s} \eta^{*}}\left(\frac{c}{N}\right)^{1 / 3} S(0) U_{0}+O\left(\frac{c}{N}\right)
$$

where $S(0)$ is the structure factor

$$
S(\mathbf{0})=n \int d V_{\mathrm{y}}[g(\mathbf{y} \mid \mathbf{0})-1]
$$

Note that $n g$ equals a delta function at $\mathbf{y}=\mathbf{0}$. For the hardsphere dispersion, $S(0)$ can be estimated from the Carnahan-Stirling approximation:

$$
S(0)=\frac{(1-c)^{4}}{1+4 c+4 c^{2}-4 c^{3}+c^{4}} .
$$

Table VIII shows the sedimentation velocity as a function of $N$ for $c=0.1$. For each $N$, we enclose in parentheses the estimate of $U(\infty) / U_{0}$ obtained with the use of (94). These estimates are essentially independent of $N$ justifying thereby the above extrapolation scheme. The correction obtained in this manner is slightly smaller than that obtained by Ladd ${ }^{11}$ who argued that there is an apparent reduction in the radius of the particles, rather than an apparent reduction in the force on the periodic images by a factor $S(0)$. He estimated this reduction in the radius of

TABLE IX. The effective viscosity $\eta^{*} / \eta$ of random suspensions of spherical drops. $\beta$ is the ratio of viscosities of the drops and the suspending fluid.

\begin{tabular}{lcccc}
\hline \hline$c \backslash \beta$ & $\infty$ & 10 & 1 & 0 \\
\hline 0.1 & 1.31 & 1.29 & 1.20 & 1.11 \\
0.25 & 2.20 & 2.07 & 1.65 & 1.32 \\
0.35 & 3.14 & 2.85 & 2.05 & 1.48 \\
0.45 & 5.4 & 4.63 & 2.81 & 1.73 \\
\hline
\end{tabular}


TABLE X. The sedimentation velocity $U / U_{0}$ of random suspensions of drops with $N=16$. The extrapolated values for $N=\infty$ are enclosed in parentheses.

\begin{tabular}{lcccc}
\hline \hline$c \backslash \beta$ & $\infty$ & 10 & 1 & 0 \\
\hline 0.1 & $0.412(0.53)$ & $0.426(0.54)$ & $0.488(0.61)$ & $0.560(0.69)$ \\
0.25 & $0.175(0.20)$ & $0.190(0.22)$ & $0.250(0.29)$ & $0.319(0.36)$ \\
0.35 & $0.086(0.096)$ & $0.098(0.11)$ & $0.146(0.16)$ & $0.203(0.22)$ \\
0.45 & $0.042(0.045)$ & $0.051(0.054)$ & $0.088(0.093)$ & $0.130(0.14)$ \\
\hline
\end{tabular}

the particles in a somewhat ad hoc manner to equal $U(\infty) / U_{0}$. At $c=0.45, S(0)$ is roughly half the correction factor $U(\infty) / U_{0}$ used by Ladd and this results in a slight difference in the estimates of sedimentation velocities.

\section{B. Drops and bubbles}

For the suspension of drops and bubbles, the problem of determining permeability is not meaningful, and therefore we only give the results for the effective viscosity and sedimentation velocity. Results are given in Tables IX and $X$. Here, $\beta$ is the ratio of viscosity of drops to the suspending fluid. The results for $\beta=\infty$ correspond to the rigid particles' case and are in good agreement with those of Ladd. The extrapolated values of the sedimentation velocity were obtained in the same manner as for the suspensions of porous particles.
Unlike the case of porous particles, the sedimentation velocity and effective viscosity deviate significantly from the corresponding values at $c=0$ in the limit of $\beta \rightarrow 0$ for an obvious reason. For porous particles, most of the flow occurs through the highly porous particles, and consequently, the particle interactions effect become weak, which is not the case for drops and bubbles.

\section{ACKNOWLEDGMENTS}

This work was supported by the National Science Foundation under Grant Nos. CTS-9118675 and CTS9307723. The authors are grateful to the Cornell Theory Center for the use of supercomputer facilities and to Professor Goddard for providing the computer program for generating packed stable arrays.

\section{APPENDIX A: EXPRESSIONS FOR THE REGULAR PARTS OF $\chi$ AND $\phi$}

\section{The regular parts of $\chi$}

Substituting for vorticity from (23) into (43) and writing $\mathscr{G}=\mathscr{G}^{T}+\mathscr{G}^{P}$ we obtain

$$
T_{k l}^{\alpha, r}=\frac{\epsilon_{k l}}{k(k+1)} \mathscr{D}_{k}^{l}\left(\mathbf{r} \cdot\left(\nabla \times \mathbf{u}^{\infty}\right)\right)(0)+T_{k l}^{\alpha, r, T}+T_{k l}^{\alpha, r, P},
$$

where $\quad T_{k l}^{\alpha, r, T}$, the contribution from $\mathbf{r} \cdot \nabla \times\left(\Sigma \mathscr{G}^{\gamma, T}\right) S_{1}\left(\mathbf{x}-\mathbf{x}^{\gamma}\right)$, is given by

$$
\begin{aligned}
T_{k l}^{\alpha, r, T}= & \frac{\epsilon_{k l}}{k(k+1)} \sum_{n, m, \gamma} \frac{1}{\lambda_{n m}}\left(-n\left(k+l \delta_{m 0}\right)\left(T_{n m}^{\gamma} \mathscr{D}_{n+k}^{m+l}+\widetilde{T}_{n m}^{\gamma} \widetilde{\mathscr{D}}_{n+k}^{m+l}\right)-(n k-n l-m l)\left(T_{n m}^{\gamma} \mathscr{D}_{n+k-2 l}^{m-l}+\widetilde{T}_{n m}^{\gamma} \widetilde{\mathscr{D}}_{n+k-2 l}^{m-l}\right)\right. \\
& \left.\times \partial_{\xi \eta}^{2 l}+\frac{l}{4}(n+m)\left(1-\delta_{m 0}\right)\left(T_{n m}^{\gamma} \mathscr{D}_{n+k-2 l+2}^{m-l}+\widetilde{T}_{n m}^{\gamma} \widetilde{\mathscr{D}}_{n+k-2 l+2}^{m-l}\right) \partial_{\xi \eta}^{2 l-2}+4 \delta_{k 1} \delta_{n 1} \delta_{l 0} \delta_{m 0} T_{10}^{\gamma} \nabla^{2}\right) S_{1}\left(\mathbf{x}^{\alpha}-\mathbf{x}^{\gamma}\right)
\end{aligned}
$$

and, in a similar manner,

$$
\begin{aligned}
T_{k l}^{\alpha, r, P}= & \frac{\epsilon_{k l}}{k(k+1)} \sum_{n, m, \gamma} \frac{1}{n \lambda_{n m}}\left[-\left(m k-n l-\frac{n l(n-1) \delta_{m 0}}{2 n-1}\right)\left(\widetilde{P}_{n m}^{\gamma} \mathscr{D}_{n+k-1}^{m+l}-P_{n m}^{\gamma} \widetilde{\mathscr{D}}_{n+k-1}^{m+l}\right)-\left(m(k-l)+\frac{l\left(n^{2}-m^{2}\right)}{2 n-1}\right)\right. \\
& \times\left(\widetilde{P}_{n m}^{\gamma} \mathscr{\mathscr { D }}_{n+k-2 l-1}^{m-l}-P_{n m}^{\gamma} \mathscr{D}_{n+k-2 l-1}^{m-l}\right) \partial_{\xi \eta}^{2 l}+\frac{l\left(1-\delta_{m 0}\right)(n+m)(n+m-1)}{4(2 n-1)} \\
& \left.\times\left(\widetilde{P}_{n m}^{\gamma} \mathscr{D}_{n+k-2 l+1}^{m-l}-P_{n m^{2}}^{\gamma} \mathscr{D}_{n+k-2 l+1}^{m-l}\right) \partial_{\xi \eta}^{2 l-2}\right] S_{1}\left(\mathbf{x}^{\alpha}-\mathbf{x}^{\gamma}\right) .
\end{aligned}
$$

The expression for $\widetilde{T}_{k l}^{\alpha, r}$ is obtained by replacing $\mathscr{D}_{k}^{l}$ in (97) by $\widetilde{\mathscr{D}}_{k}^{l}$ and by replacing the last two terms on the right-hand side of the same equation by corresponding quantities with tilde defined by

$$
\begin{aligned}
\tilde{T}_{k l}^{\alpha, r}, T= & \frac{\epsilon_{k l}}{k(k+1)} \sum_{n, m, \gamma} \frac{1}{\lambda_{n m}}\left(n\left(k+l \delta_{m 0}\right)\left(\tilde{T}_{n m}^{\gamma} \mathscr{\mathscr { D }}_{n+k}^{m+l}-T_{n m}^{\gamma} \widetilde{\mathscr{D}}_{n+k}^{m+l}\right)-(n k-n l-m l)\left(\tilde{T}_{n m}^{\gamma} \mathscr{D}_{n+k-2 l}^{m-l}-T_{n m}^{\gamma} \widetilde{\mathscr{D}}_{n+k-2 l}^{m-l}\right) \partial_{\xi \eta}^{2 l}\right. \\
& \left.+\frac{l}{4}(n+m)\left(1-\delta_{m 0}\right)\left(\tilde{T}_{n m}^{\gamma} \mathscr{\mathcal { D }}_{n+k-2 l+2}^{m-l}-T_{n m}^{\gamma} \widetilde{\mathscr{W}}_{n+k-2 l+2}^{m-l}\right) \partial_{\xi \eta}^{2 l-2}\right) S_{1}\left(\mathbf{x}^{\alpha}-\mathbf{x}^{\gamma}\right)
\end{aligned}
$$

and 


$$
\begin{aligned}
\tilde{T}_{k l}^{\alpha, r, P}= & \frac{\epsilon_{k l}}{k(k+1)} \sum_{n, m, \gamma} \frac{1}{n \lambda}\left[-\left(m k-n l-\frac{n l(n-1) \delta_{m 0}}{2 n-1}\right)\left(P_{n m^{\prime}}^{\gamma} \mathscr{D}_{n+k-1}^{m+l}+\widetilde{P}_{n m}^{\gamma} \mathscr{D}_{n+k-1}^{m+l}\right)+\left(m(k-l)+\frac{l\left(n^{2}-m^{2}\right)}{2 n-1}\right)\right. \\
& \times\left(P_{n m}^{\gamma} \mathscr{D}_{n+k-2 l-1}^{m-l}+\widetilde{P}_{n m}^{\gamma} \mathscr{\mathscr { D }}_{n+k-2 l-1}^{m-l}\right) \partial_{\xi \eta}^{2 l}-\frac{l\left(1-\delta_{m 0}\right)(n+m)(n+m-1)}{4(2 n-1)} \\
& \left.\times\left(P_{n m}^{\gamma} \mathscr{D}_{n+k-2 l+1}^{m-l}+\widetilde{P}_{n m}^{\gamma} \mathscr{D}_{n+k-2 l+1}^{m-l}\right) \partial_{\xi \eta}^{2 l-2}\right] S_{1}\left(\mathbf{x}^{\alpha}-\mathbf{x}^{\gamma}\right) .
\end{aligned}
$$

In obtaining the above relations the intermediate step typically involved products of two differential operators such as $\mathscr{D}_{k}^{l} \mathscr{D}_{n}^{m}$. The following relations were used to convert them to a sum of differential operators:

$$
\Delta_{m} \Delta_{l}=\left(\partial_{\xi}^{m}+\partial_{\eta}^{m}\right)\left(\partial_{\xi}^{l}+\partial_{\eta}^{l}\right)=\Delta_{m+l}+\partial_{\xi \eta}^{2 l} \Delta_{m-l},
$$

with

$$
\partial_{\xi \eta}^{2 l}=2^{-2 l}\left(\nabla^{2}-\partial_{1}^{2}\right)^{l}
$$

For $l>m$, the last term in (102) may alternatively be expressed as $\partial_{\xi \eta}^{2 m} \Delta_{l-m}$.

\section{The regular parts of $\phi$}

Since $\psi$ can be expressed as $\mathbf{r} \cdot \mathbf{u}^{\infty}+\mathbf{r} \cdot \mathscr{G}^{\alpha, T} S_{1}+\mathbf{r} \cdot \mathscr{G}^{\alpha, P} S_{1}$

$$
+\mathbf{r} \cdot \nabla\left(\mathscr{H}^{\alpha} S_{1}-\left(\nabla \cdot \mathscr{G}^{\alpha}\right) S_{2}\right)
$$

we write

$$
\Phi_{k l}^{\alpha, r}=\epsilon_{k l} k^{-1} \mathscr{D}_{k}^{l}\left(\mathbf{r} \cdot \mathbf{u}^{\infty}\right)(0)+\Phi_{k l}^{\alpha, r, G T}+\Phi_{k l}^{\alpha, r, G P}+\Phi_{k l}^{\alpha, r, \Psi}
$$

where the four terms on the right-hand side correspond to the four terms of (A8). Substituting each of the terms from (A8) into (A6), and using (102) for $\mathscr{D}_{k}^{l} \mathscr{D}_{n}^{m}$, etc., we obtain the following expressions for the above quantities:

$$
\begin{aligned}
& \Phi_{k l}^{\alpha, r, G T}=\frac{\epsilon_{k l}}{k} \sum_{n, m, \gamma} \frac{1}{\lambda_{n m}}\left(-\left(m k-n l\left(1+\delta_{m 0}\right)\right)\left(\widetilde{T}_{n m}^{\gamma} \mathscr{D}_{n+k-1}^{m+l}-T_{n m}^{\gamma} \widetilde{\mathscr{D}}_{n+k-1}^{m+l}\right)-m(k-l)\left(\tilde{T}_{n m}^{\gamma} \mathscr{D}_{n+k-2 l-1}^{m-l}\right.\right. \\
& \left.\left.-T_{n m}^{\gamma} \widetilde{\mathscr{D}}_{n+k-2 l-1}^{m-l}\right) \partial_{\xi \eta}^{2 l}+\frac{l}{4}\left(1-\delta_{m 0}\right)(n+m)\left(\widetilde{T}_{n m}^{\gamma} \mathscr{D}_{n+k-2 l+1}^{m-l}-T_{n m}^{\gamma} \widetilde{\mathscr{D}}_{n+k-2 l+1}^{m-l}\right) \partial_{\xi \eta}^{2 l-2}\right) S_{1}\left(\mathbf{x}^{\alpha}-\mathbf{x}^{\gamma}\right), \\
& \widetilde{\Phi}_{k l}^{\alpha, r}, G T=\frac{\epsilon_{k l}}{k} \sum_{n, m, \gamma} \frac{1}{\lambda_{n m}}\left(-\left(m k-n l\left(1+\delta_{m 0}\right)\right)\left(T_{n m}^{\gamma} \mathscr{D}_{n+k-1}^{m+l}+\widetilde{T}_{n m}^{\gamma} \mathscr{\mathscr { D }}_{n+k-1}^{m+l}\right)+m(k-l)\left(T_{n m}^{\gamma} \mathscr{D}_{n+k-2 l-1}^{m-l}\right.\right. \\
& \left.\left.+\widetilde{T}_{n m}^{\gamma} \widetilde{\mathscr{D}}_{n+k-2 l-1}^{m-l}\right) \partial_{\xi \eta}^{2 l}-\frac{l}{4}\left(1-\delta_{m 0}\right)(n+m)\left(T_{n m}^{\gamma} \mathscr{D}_{n+k-2 l+1}^{m-l}+\widetilde{T}_{n m}^{\gamma} \widetilde{\mathscr{D}}_{n+k-2 l+1}^{m-l}\right) \partial_{\xi \eta}^{2 l-2}\right) S_{1}\left(\mathbf{x}^{\alpha}-\mathbf{x}^{\gamma}\right) \\
& \Phi_{k l}^{a, r, G P}=\frac{\epsilon_{k l}}{k} \sum_{n, m, \gamma} \frac{1}{2 n(2 n-1) \lambda_{n m}}\left(2(n-m)\left(l(n-m-1)\left(1+\delta_{m 0}\right)-(k-l)(n+m)\right)\left(P_{n m}^{\gamma} \mathscr{D}_{n+k-2}^{m+l}+\widetilde{P}_{n m}^{\gamma} \widetilde{D}_{n+k-2}^{m+l}\right)\right. \\
& -2(k-l)(n+m)(n-m)\left(P_{n m}^{\gamma} \mathscr{D}_{n+k-2 l-2}^{m-l}+\widetilde{P}_{n m}^{\gamma} \widetilde{D}_{n+k-2 l-2}^{m-l}\right) \partial_{\xi \eta}^{2 l}-\frac{l}{2}\left(1-\delta_{m 0}\right)(n+m)(n+m-1) \\
& \left.\times\left(P_{n m}^{\gamma} \mathscr{D}_{n+k-2 l}^{m-l}+\tilde{P}_{n m}^{\gamma} \mathscr{D}_{n+k-2 l}^{m-l}\right) \partial_{\xi \eta}^{2 l-2}+\frac{2}{5} \delta_{k 3} \delta_{n 1}\left(12 \delta_{k 0} \delta_{m 0} P_{10}^{\gamma}+\delta_{l 1} \delta_{m 1} P_{11}^{\gamma}\right) \nabla^{2}\right) S_{1}\left(\mathbf{x}^{\alpha}-\mathbf{x}^{\gamma}\right), \\
& \widetilde{\Phi}_{k l}^{\alpha, r, G P}=\frac{\epsilon_{k l}}{k} \sum_{n, m, \gamma} \frac{\lambda_{n m}^{-1}}{2 n(2 n-1)}\left(-2(n-m)\left(l(n-m-1)\left(1+\delta_{m 0}\right)-(k-l)(n+m)\right)\left(\widetilde{P}_{n m}^{\gamma} \mathscr{D}_{n+k-2}^{m+l}-P_{n m}^{\gamma} \widetilde{\mathscr{D}}_{n+k-2}^{m+l}\right)\right. \\
& +2(k-l)(n+m)(n-m)\left(\widetilde{P}_{n m}^{\gamma} \mathscr{D}_{n+k-2 l-2}^{m-l}-P_{n m}^{\gamma} \widetilde{D}_{n+k-2 l-2}^{m-l}\right) \partial_{\xi \eta}^{2 l}-\frac{l}{2}\left(1-\delta_{m 0}\right)(n+m)(n+m-1) \\
& \left.\times\left(\widetilde{P}_{n m}^{\gamma} \mathscr{D}_{n+k-2 l}^{m-l}-P_{n m}^{\gamma} \mathscr{D}_{n+k-2 l}^{m-l}\right) \partial_{\xi \eta}^{2 l-2}+\frac{2}{5} \delta_{k 3} \delta_{n 1} \delta_{l 1} \delta_{m 1} \widetilde{P}_{11}^{\gamma} \nabla^{2}\right) S_{1}\left(\mathbf{x}^{\alpha}-\mathbf{x}^{\gamma}\right),
\end{aligned}
$$




$$
\begin{aligned}
& \Phi_{k l}^{\alpha, r, \Psi}=\epsilon_{k l} \sum_{n, m, \gamma} \lambda_{n m}^{-1}\left[\left(\mathscr{D}_{n+k}^{m+l}+\mathscr{D}_{n+k-2 l}^{m-l} \partial_{\xi \eta}^{2 l}\right)\left(\lambda_{n m} H_{n m}^{\gamma} S_{1}+P_{n m}^{\gamma} S_{2}\right)+\left(\widetilde{\mathscr{D}}_{n+k}^{m+l}+\widetilde{\mathscr{D}}_{n+k-2 l}^{m-l} \partial_{\xi \eta}^{2 l}\right)\left(\lambda_{n m} \tilde{H}_{n m}^{\gamma} S_{1}+\tilde{P}_{n m}^{\gamma} S_{2}\right)\right. \\
& -\frac{n-m}{2}\left(\left(\mathscr{D}_{n+k-1}^{m+l}+\mathscr{D}_{n+k-2 l-1}^{m-l} \partial_{\xi \eta}^{2 l}\right) \widetilde{T}_{n m}^{\gamma}-\left(\widetilde{\mathscr{D}}_{n+k-1}^{m+l}+\widetilde{\mathscr{D}}_{n+k-2 l-1}^{m-l} \partial_{\xi \eta}^{2 l}\right) T_{n m}^{\gamma}\right) S_{1} \\
& -\left(\frac{(n-m)(n-m-1)\left(1+\delta_{m 0}\right)}{2 n(2 n-1)}+\frac{(k-2)(k-l)(k-l-1)}{2 k(2 k-1)}\right)\left(\left(\mathscr{D}_{n+k-2}^{m+l}+\mathscr{D}_{n+k-2 l-2}^{m-l} \partial_{\xi \eta}^{2 l}\right) P_{n m}^{\gamma}+\left(\mathscr{D}_{n+k-2}^{m+l}\right.\right. \\
& \left.\left.\left.+\widetilde{\mathscr{D}}_{n+k-2 l-2}^{m-l} \partial_{\xi \eta}^{2 l}\right) \widetilde{P}_{n m}^{\gamma}\right) S_{1}\right] \\
& \tilde{\Phi}_{k l}^{\alpha, r},=\epsilon_{k l} \sum_{n, m, \gamma} \lambda_{n m}^{-1}\left[-\left(\mathscr{D}_{n+k}^{m+l}-\mathscr{D}_{n+k-2 l}^{m-l} \partial_{\xi \eta}^{2 l}\right)\left(\lambda_{n m} \tilde{H}_{n m}^{\gamma} S_{1}+\widetilde{P}_{n m}^{\gamma} S_{2}\right)+\left(\mathscr{D}_{n+k}^{m+l}-\widetilde{\mathscr{D}}_{n+k-2 l}^{m-l} \partial_{\xi \eta}^{2 l}\right)\left(\lambda_{n m} H_{n m}^{\gamma} S_{1}+P_{n m}^{\gamma} S_{2}\right)\right. \\
& -\frac{n-m}{2}\left(\left(\mathscr{D}_{n+k-1}^{m+l}-\mathscr{D}_{n+k-2 l-1}^{m-l} \partial_{\xi \eta}^{2 l}\right) T_{n m}^{\gamma}+\left(\mathscr{D}_{n+k-1}^{m+l}-\mathscr{D}_{n+k-2 l-1}^{m-l} \partial_{\xi \eta}^{2 l}\right) \tilde{T}_{n m}^{\gamma}\right) S_{1} \\
& +\left(\frac{(n-m)(n-m-1)\left(1+\delta_{m 0}\right)}{2 n(2 n-1)}+\frac{(k-2)(k-l)(k-l-1)}{2 k(2 k-1)}\right)\left(\left(\mathscr{D}_{n+k-2}^{m+l}-\mathscr{D}_{n+k-2 l-2}^{m-l} \partial_{\xi \eta}^{2 l}\right) \widetilde{P}_{n m}^{\gamma}-\left(\mathscr{D}_{n+k-2}^{m+l}\right.\right. \\
& \left.\left.\left.-\mathscr{D}_{n+k-2 l-2}^{m-l} \partial_{\xi \eta}^{2 l}\right) P_{n m}^{\gamma}\right) S_{1}\right],
\end{aligned}
$$

where $S_{1}$ and $S_{2}$ and their derivatives are evaluated at $\mathbf{x}^{\alpha}-\mathbf{x}^{\gamma}$.

\section{APPENDIX B: EVALUATION OF $\lambda_{1}$ AND $\lambda_{2}$}

\section{Evaluation of $\lambda_{1}$}

To compute the integral of $\omega^{2}$ on the surface of particle $\alpha$, we express the vorticity as a sum of poloidal and toroidal fields: ${ }^{18}$

$$
\omega=\nabla \times \mathbf{u}=\mathbf{T}+\mathbf{S},
$$

with

$$
\begin{aligned}
& \mathbf{T}=\nabla \times\left(\frac{\mathscr{T}}{r} \mathbf{r}\right), \quad \mathscr{T}=\sum_{n=-\infty}^{\infty}\left(b_{n}-2 c_{n}\right) r p_{n}, \\
& \mathbf{S}=\nabla \times \nabla \times\left(\frac{\mathscr{S}}{r} \mathbf{r}\right), \quad \mathscr{S}=\sum_{n=-\infty}^{\infty} \chi_{n} .
\end{aligned}
$$

Here, $\mathscr{T}$ and $\mathscr{S}$ are the scalar functions defining the toroidal and poloidal fields, $\mathbf{r}=\mathbf{x}-\mathbf{x}^{\alpha}$, and $c_{n}$ and $b_{n}$ defined via (7)-(8). The above expansion for the vorticity is valid near the surface of particle $\alpha$. Note that, for brevity, we have suppressed superscript $\alpha$ on $p_{n}$ and $\chi_{n}$. Now using the orthogonal properties of the toroidal and poloidal fields ( $p$. 625 , Ref. 18), we obtain

$$
\begin{aligned}
\int_{S^{\alpha}} \omega^{2} d A= & \sum_{n=1}^{\infty} \sum_{m=0}^{n} \Lambda_{n m}\left[d_{n m}^{2}+n(n+1) a^{-2} e_{n m}^{2}\right. \\
& \left.+\left(\frac{d e_{n m}}{d r}\right)^{2}+\text { tilde terms }\right]_{r=a},
\end{aligned}
$$

where

$$
\begin{aligned}
\sum_{n=-\infty}^{\infty}\left(b_{n}-2 c_{n}\right) r p_{n}= & \sum_{n=1}^{\infty} \sum_{m=0}^{n}\left[d_{n m}(r) \cos m \varphi\right. \\
& \left.+\tilde{d}_{n m}(r) \sin m \varphi\right] P_{n}^{n}(\cos \theta)
\end{aligned}
$$

$$
\begin{gathered}
\sum_{n=-\infty}^{\infty} r \chi_{n}=\sum_{n=1}^{\infty} \sum_{m=0}^{n}\left[e_{n m}(r) \cos m \varphi\right. \\
\left.\quad+\widetilde{e}_{n m}(r) \sin m \varphi\right] P_{n}^{m}(\cos \theta), \\
\Lambda_{n m}=\frac{2 \pi n(n+1)(n+m) !}{(2 n+1)(n-m) !}\left(1+\delta_{m 0}\right) .
\end{gathered}
$$

Now, noting that $b_{n}-2 c_{n}=-1 /(n+1)$, we express $d_{n m}$ and $e_{n m}$ in terms of $P_{n m}$, etc., by

$$
\begin{aligned}
& d_{n m}(r)=\frac{1}{n} P_{n m} r^{-n}-\frac{1}{n+1} P_{n m}^{r} r^{n+1}, \\
& e_{n m}(r)=T_{n m} r^{-n}+T_{n m}^{r} r^{n+1} .
\end{aligned}
$$

Using (48)-(50) we relate the regular term coefficients, $P_{n m}^{r}$ and $T_{n m}^{r}$ to $P_{n m}$, etc., and substituting for $d_{n m}, e_{n m}$, and their derivatives into (B4) we obtain

$$
\begin{aligned}
\int_{S^{\alpha}} \omega^{2} d A= & \sum_{n=1}^{\infty} \sum_{m=0}^{n} \Lambda_{n m}(2 n+1)^{2} a^{-2 n}\left[P_{n m}^{* 2}\right. \\
& \left.+a^{-2} T_{n m}^{2}+\text { tilde terms }\right],
\end{aligned}
$$

where

$$
P_{n m}^{*}=-\frac{1}{2 n} P_{n m}+\frac{2 n+3}{n a^{2}} \Phi_{n m}
$$


Thus, the surface integral of $\omega^{2}$ on each particle is related to the multipoles through a relatively simple expression.

\section{Evaluation of $\lambda_{2}$}

Let us take, without loss of generality, $\mathbf{U}^{\infty}$ to be along the $x_{1}$ axis and evaluate the component of (88) along the $x_{1}$-axis. Using $v_{11}=S_{1}-\partial^{2}{ }_{11} S_{2}=\left(\partial_{22}^{2}+\partial_{33}^{2}\right) S_{2}$, and converting the volume integrals in (88) to the surface integrals we obtain

$$
\begin{aligned}
\int_{V^{\alpha}} & \int_{V^{\gamma}} v_{11}(\mathbf{x}-\mathbf{y}) d V_{\mathbf{x}}^{\alpha} d V_{\mathbf{y}}^{\gamma} \\
= & -\int_{S^{\alpha}} \int_{S^{\gamma}}\left(n_{2}(\mathbf{x}) n_{2}(\mathbf{y})+n_{3}(\mathbf{x}) n_{3}(\mathbf{y})\right) \\
& \times S_{2}(\mathbf{x}-\mathbf{y}) d A_{\mathbf{x}}^{\alpha} d A_{\mathbf{y}}^{\gamma} .
\end{aligned}
$$

Now since $S_{2}$ is a biharmonic function, the surface integral of $n_{2} S_{2}$ equals $V_{p}\left(\partial_{2}+\frac{1}{10} \nabla^{2} \partial_{2}\right) S_{2}$ evaluated at the center of particle when $S_{2}$ is regular over the entire volume of the particle. Otherwise, the singular part of $S_{2}$, i.e., $r / 2$, must first be removed and the integration of regular and singular parts be carried separately. On further using $\nabla^{2} S_{2}=S_{1}$, and simplifying, the above surface integrals reduce to

$$
V_{p}^{2}\left[\frac{4}{5 a} \delta_{\alpha, \gamma}+\left(\nabla^{2}-\partial_{1}^{2}\right)\left(1+\frac{a^{2}}{5} \nabla^{2}\right) S_{2}\left(\mathbf{x}^{\alpha}-\mathbf{x}^{\gamma}\right)\right],
$$

where $V_{p}=4 \pi a^{3} / 3$ is the volume of particles. Note that $S_{2}$ in the above expression must be replaced by its regular part for $\alpha=\gamma$. The first term in the above expression corresponds to the contribution from the singular part and must cancel with the corresponding term in the expansion of $F_{0}$, the force on an isolated particle so that on simplifying we obtain

$$
\lambda_{2}=\frac{a}{3 N} \sum_{\alpha=1}^{N} \sum_{\gamma=1}^{N}\left(\nabla^{2}-\partial^{2}{ }_{1}\right)\left(1+\frac{a^{2}}{5} \nabla^{2}\right) S_{2}\left(\mathbf{x}^{\alpha}-\mathbf{x}^{\gamma}\right)
$$

For cubic arrays with $N=1$, this yields

$$
\lambda_{2}=\frac{2}{9}\left[a S_{1}(0)+\frac{3 c}{5}\right] \text {. }
$$

${ }^{1}$ P. Ganatos, R. Pfeffer, and S. Weinbaum, "A numerical-solution technique for three-dimensional Stokes flow, with application to the motion of strongly interacting spheres in a plane," J. Fluid Mech. 84, 79 (1978).

${ }^{2} \mathrm{P}$. Mazur and W. van Saarloos, "Many-sphere hydrodynamic interactions and mobilities in a suspension," Physica A 115, 21 (1982).

${ }^{3}$ W. van Saarloos and P. Mazur, "Many-sphere hydrodynamic interactions. II. Mobilities at finite frequencies," Physica A 120, 77 (1983).

${ }^{4} \mathrm{C}$. W. J. Beenakker, "The effective viscosity of a concentrated suspension of spheres (and its relation to diffusion)," Physica A 128, 48 (1984).

${ }^{5} \mathrm{C}$. W. J. Beenakker and P. Mazur, "Diffusion in a concentrated suspension II," Physica A 126, 349 (1984).

${ }^{6} \mathrm{G}$. Bossis and J. F. Brady, "Dynamic simulation of sheared suspensions. I. General method," J. Chem. Phys. 80, 5141 (1984).
${ }^{7}$ L. Durlofsky, J. F. Brady, and G. Bossis, "Dynamic simulation of hydrodynamically interacting particles," J. Fluid Mech. 180, 21 (1987).

${ }^{8}$ J. F. Brady and G. Bossis, "Stokesian dynamics," Annu. Rev. Fluid Mech. 20, 111 (1988).

${ }^{9}$ A. J. C. Ladd, "Hydrodynamic interactions in a suspension of spherical particles," J. Chem. Phys. 88, 5051 (1988).

${ }^{10} \mathrm{~A}$. J. C. Ladd, "Hydrodynamic interactions and the viscosity of suspensions of freely moving spheres," J. Chem. Phys. 90, 1149 (1989).

"A. J. C. Ladd, "Hydrodynamic transport coefficients of random dispersions of hard spheres," J. Chem. Phys. 93, 3484 (1990).

${ }^{12} \mathrm{H}$. Hasimoto, "On the periodic fundamental solutions of the Stokes equations and their application to viscous flow past a cubic array of spheres," J. Fluid Mech. 5, 317 (1959).

${ }^{13}$ H. Lamb, Hydrodynamics (Dover, New York, 1945).

${ }^{14} \mathrm{~J}$. Happel and H. Brenner, Low Reynolds Number Hydrodynamics (Martinus Nijhoff, Dordrecht, 1986).

${ }^{15} \mathrm{E}$. W. Hobson, The Theory of Spherical and Ellipsoidal Harmonics (Cambridge University Press, Cambridge, 1931).

${ }^{16} \mathrm{~A}$. S. Sangani and A. Acrivos, "Slow flow through a periodic array of spheres," Int. J. Multiphase Flow 8, 343 (1982).

${ }^{17}$ A. S. Sangani and C. Yao, "Bulk conductivity of composites with spherical inclusions," J. Appl. Phys. 63, 1334 (1988).

${ }^{18} \mathrm{~S}$. Chandrasekhar, Hydrodynamic and Hydromagnetic Stability (Dover, New York, 1981).

${ }^{19}$ S.-Y. Kang and A. S. Sangani, "Electrokinetic properties of suspensions of colloidal particles with thin, polarized double layers," to appear in J. Colloid Interface Sci. (1994).

${ }^{20} \mathrm{G}$. S. Beavers and D. D. Joseph, "Boundary conditions at a naturally permeable wall,” J. Fluid Mech. 30, 197 (1967).

${ }^{21}$ P. G. Saffman, "On the boundary conditions at the surface of a porous medium," Stud. Appl. Math. 2, 93 (1971).

${ }^{22}$ J. J. L. Higdon and M. Kojima, "On the calculation of Stokes' flow past porous particles," Int. J. Multiphase Flow 7, 719 (1981).

${ }^{23}$ R. E. Larson and J. J. L. Higdon, "Microscopic flow near the surface of two-dimensional porous media. Part 1. Axial flow," J. Fluid Mech. 166, 449 (1986).

${ }^{24}$ A. S. Sangani and S. Behl, "The planar singular solutions of Stokes and Laplace equations and their application to transport processes near porous surfaces," Phys. Fluids A 1, 21 (1989).

${ }^{25} \mathrm{M}$. Abramowitz and I. A. Stegun, Handbook of Mathematical Functions with Formulas, Graphs, and Mathematical Tables (National Bureau of Standards, Washington, DC, 1972).

${ }^{26} \mathrm{~S}$. Kim and S. J. Karrila, Microhydrodynamics (ButterworthHeinemann, Boston, 1991).

${ }^{27}$ A. S. Sangani, D. Z. Zhang, and A. Prosperetti, "The added mass, Basset, and viscous drag coefficients in nondilute bubbly liquids undergoing small-amplitude oscillatory motion," Phys. Fluids A 3, 2955 (1991).

${ }^{28}$ J. D. Goddard, X. Zhuang, and A. K. Didwania, "Microcell methods and the adjacency matrix in the simulation of the mechanics of granular media," in Proceedings of the 2nd International Conference on the Discrete Element Methods, edited by J. R. Williams and G. G. W. Mustoe [IESL Publications (MIT), Cambridge, MA, 1993].

${ }^{29} \mathrm{P}$. Debye and A. M. Bueche, "Intrinsic viscosity, diffusion, and sedimentation rate of polymers in solution," J. Chem. Phys. 16, 573 (1948).

${ }^{30} \mathrm{~B}$. U. Felderhof, "Frictional properties of dilute polymer solutions. III. Translational-friction coefficient," Physica A 80, 63 (1975).

${ }^{31}$ P. C. Carman, Trans. Inst. Chem. Eng. 15, 150 (1937).

${ }^{32}$ A. A. Zick and G. M. Homsy, "Stokes flow through periodic arrays of spheres," J. Fluid Mech. 115, 13 (1982).

${ }^{33} \mathrm{G}$. Neale, N. Epstein, and W. Nader, "Creeping flow relative to permeable spheres," Chem. Eng. Sci. 28, 1865 (1973).

${ }^{34} \mathrm{R}$. H. Davis and H. A. Stone, "Flow through beds of porous particles," Chem. Eng. Sci. 48, 4005 (1993).

${ }^{35} \mathrm{~B}$. U. Felderhof, "Frictional properties of dilute polymer solutions. IV. Intrinsic viscosity," Physica A 80, 172 (1975).

${ }^{36}$ R. J. Phillips, J. F. Brady, and G. Bossis, "Hydrodynamic transport properties of hard-sphere dispersions. I. Suspensions of freely mobile particles," Phys. Fluids 31, 3462 (1988).

${ }^{37}$ The authors are grateful to Professor D. L. Koch for this suggestion. 